\title{
Ice formation on a smooth or rough cold surface due to the impact of a supercooled water droplet
}

\author{
J. W. Elliott (j.w.elliott@hull.ac.uk ) \\ Department of Physics \& Mathematics, University of Hull, Hull HU6 7RX, UK \\ F. T. Smith (f.smith@ucl.ac.uk ) \\ Department of Mathematics, UCL, Gower Street, London WC1E 6BT, UK
}

\begin{abstract}
Ice accretion is considered in the impact of a supercooled water droplet on a smooth or rough solid surface, the roughness accounting for earlier icing. In this theoretical investigation the emphasis and novelty lie in the full nonlinear interplay of the droplet motion and the growth of the ice surface being addressed for relatively small times, over a realistic range of Reynolds numbers, Froude numbers, Weber numbers, Stefan numbers and capillary underheating parameters. The Prandtl number and the kinetic under-heating parameter are taken to be order unity. The ice accretion brings inner layers into play forcibly, affecting the outer flow. (The work includes viscous effects in an isothermal impact without phase change, as a special case, and the differences between impact with and without freezing.) There are four main findings. First, the icing dynamically can accelerate or decelerate the spreading of the droplet whereas roughness on its own tends to decelerate spreading. The interaction between the two and the implications for successive freezings are found to be subtle. Second, a focus on the dominant physical effects reveals a multi-structure within which restricted regions of turbulence are implied. The third main finding is an essentially parabolic shape for a single droplet freezing under certain conditions. Fourth is a connection with a body of experimental and engineering work and with practical findings to the extent that the explicit predictions here for ice-accretion rates are found to agree with the experimental range.
\end{abstract}

Keywords: Ice accretion; droplets; supercooled; multi-structure

\section{Introduction}

The high-speed impact of a two-dimensional supercooled cylindrical water droplet or small drop on a dry, possibly rough, but essentially flat, solid surface is investigated in the current paper. In addition to considering the flow, which is governed by inertial, viscous, gravitational and capillary effects, characterised by the Reynolds number, the Froude number and the Weber number, we have other parameters to account for relating to the heat transfer and temperature dependence of the material properties. Moreover we have phase changes, which here are assumed to be confined to solidification of the drop, avoiding the phenomena of vaporisation and substrate melting. The fluid mechanics of drop impact with surfaces is of importance in a variety of different fields. For example high-speed impacts with solids can cause severe erosion, whilst engineering applications include spray cooling and ink-jet printing.

(C) 2015 Kluwer Academic Publishers. Printed in the Netherlands. 
Ice formation during an impact or a succession of impacts can readily lead to complex phenomena due to flow interacting with heat transfer and phase changes. This leads to our focus. A range of potentially relevant features is discussed first in the introduction below and then the focus is drawn to major aspects in anticipation of the work within the present paper.

\subsection{ICING AND IMPACTS}

A supercooled water droplet can exist because its transition to its stable solid phase first requires energy to nucleate. The formation of a microscopic amount of the solid phase necessarily involves the creation of a high free-energy interface between the two phases. In principle, in the absence of external perturbations, a water droplet can be continued to be cooled until eventually localized thermally-driven fluctuations cause so-called homogeneous nucleation [1]. In practice, and at much greater temperatures, the necessary energy is provided by impurity particles (dust) or by contact with a solid object, socalled heterogeneous nucleation, albeit the droplet is still supercooled by tens of degrees.

Consequently clouds may contain liquid water of several grams per cubic metre in the form of supercooled droplets for prolonged periods. Large supercooled water droplets (typically up to a radius $2.5 \mathrm{~mm}$ ) only exist at warmer ambient temperatures but relatively small cloud droplets may frequently exist in the supercooled state down to $-20^{\circ} \mathrm{C}$ and less frequently as low as -30 to $-35^{\circ} \mathrm{C}$. Indeed very small water droplets have been observed to remain supercooled down to $-40^{0} \mathrm{C}$ [2]. Thus freezing rain, which is effectively a supercooled cloud of very large droplets, is unlikely to exist below $-20^{0} \mathrm{C}$, whereas freezing drizzle, which has droplets with smaller diameters $50-400 \mu \mathrm{m}$, could exist at lower temperatures.

Our main concern is with regard to aircraft icing [3, 4] and comparing with experiments where possible. When an aircraft is flying through clouds at an ambient temperature below freezing, supercooled water droplets suspended in the cloud can impact and accrete ice on the forward-facing parts of the aircraft, most crucially about the leading edge of a wing, on the tail and around the engine intakes. Ice build-up can have a significant impact on the aircraft aerodynamics, through loss of lift and increase in drag, possibly leading to loss of life. Usually such areas are equipped with de-icing technology such as using warm bleed air from engine compressors or forcing freezing point depressant fluid (normally glycerine and water) out of porous panels. Although such measures can evaporate impinging water droplets or melt accreted ice, there is the potential risk of runback ice forming further downstream due to a refreezing of the ice-water mixture on unprotected areas.

Recent studies on the freezing of water droplets indicate a two-stage freezing process, a fast recalescence stage in which supercooling drives ki- 
netic crystal growth followed by a slower stage governed by the heat transfer rate [5]. For a droplet placed on a flat solid surface [6] identify the fast stage with a moving front rather than an instantaneous occurrence. Moreover, for superhydrophobic dry (unsaturated flow) surfaces with a sufficiently strong shear gas flow, this fast stage can emanate from a homogeneous nucleation at the upstream gas/liquid interface. Finally here [7] show that supercooled droplets impacting on inclined, exceptionally superhydrophobic, dry surfaces can bounce-off without freezing.

However, for a humid environment and a surface that is in general contaminated by ice crystals, impacting droplets will freeze either partially or completely, from a heterogeneous nucleation at the solid/liquid interface. The latent heat of fusion released from the water which freezes, and which tends to warm up the impacted surface, is countered by the convective heat loss to the ambient air. For cold ambient temperatures, low airspeed and low liquid water content (LWC) the droplets freeze completely on impact. They tend to form a white opaque accretion, called rime ice, which is relatively streamlined but with a much greater surface roughness than the wing. At ambient temperatures closer to freezing, at high airspeeds, or for clouds with high LWC, only part of the liquid water freezes on impact, the remainder running aft along the surface. It is possible for this water to freeze at aft locations, producing localized thickening of the ice profile, giving rise to horned ice shapes, on both the upper and lower surfaces. This accretion tends to be translucent in appearance and is termed glaze ice. Other possible reasons for the formation of ice further downstream on the wing may be due to splashing and reentrainment of droplets into the air flow. Another key issue is the unknown role of surface roughness caused say by previous freezings and its interplay with ice growth, in the overall droplet dynamics.

The impact of a single, initially spherical, liquid droplet or drop with a surface has received much attention since the studies of [8] and [9]. The behaviour of a liquid droplet after impact depends on whether the surface is hydrophobic or hydrophillic, rough or smooth and whether it is solid or liquid. Even for the case of a dry solid smooth horizontal plane surface and a normal impact velocity there are several stages [10] resulting in either bouncing, spreading or splashing. The initial kinematic compressive stage, where the drop resembles a truncated sphere, appears to depend only on the impact velocity and diameter. This is followed by a spreading stage where a thin jet (lamella) is ejected from the base of the drop forming a rapidly expanding thinning film bounded by a thicker rim. During this stage the drop changes from a sphere to a disc-like shape and is increasingly subject to viscous forces and, to a lesser extent, influenced by surface tension, surface roughness and wettability. For a sufficiently large impact speed the drop will splash, which includes separation of tiny drops from the wave crests of the unstable rim (prompt splash) but usually refers to detachment of the lamella 
from the surface where it forms a crown from which tiny droplets are ejected (corona splash). See [11] concerning splashes as well as parametric roughness effects. For low to moderate impact velocities, at least in the non-wetting case, the contact diameter attains and decreases from a maximum, dewetting the surface. This recoil can either be sufficiently strong to cause the drop to bounce off the surface or else the drop equilibrates to some constant diameter, possibly after further expansions and contractions. Finally we should mention [12]'s experiments on impacts onto extremely inclined surfaces, in which asymmetry and sliding enter the problem.

Most theoretical investigations of drop impact focus on the later spreading stage of the thinning liquid film and are concerned with predicting the maximum spreading diameter or the splashing threshold. For comprehensive reviews of the subject see [13], [14]. Following [15] the usual approach is an energy balance equating the known energy of the impacting drop with that of the lamella (kinetic, potential, surface and dissipated energy). To obtain the spread factor as a function of time the estimate for the kinetic energy requires assumptions for both the velocity field and the drop shape. However at maximum spread, where the kinetic energy is minimal or comparatively low, only the dissipated energy is not well-defined and requires approximation. Typical of this approach are the studies by [16], [17], [18], [19] in which simple algebraic expressions for the maximum spreading diameter in terms of the impact Weber and Reynolds numbers are obtained. In contrast, [20], [21], and [22] argue that the conventional energy balance fails to take into account the substantial volume of fluid in the rim. They contend that, for an inertia dominated impact at high Reynolds and Weber numbers, the evolution of the expanding lamella in later stages of spreading is described by a universal flow. This, in contrast to the above, leads to predictions for the residual film thickness and the maximum spreading diameter independent of the impact parameters. See also [23]. Recently, [24] extended the analysis to cover heat transfer and phase transition by finding a self-similar solution to the NavierStokes and energy equations, albeit for the case of equal densities and heat capacities for both phases.

A link with the freezing of supercooled droplets is provided by the theoretical study of the impact and solidification of liquid metal drops on cooled substrates. This is also a highly complicated solidification free-surface problem, albeit that the liquid is superheated rather than supercooled. The energy-balance approach of [15] was adapted by [25], [26] to yield a simple splat-quench solidification model (see also [27] and [28]). In order to improve the agreement with experiment for impact problems involving rapid solidification (large Stefan number), some investigators ([29]) have used undercooling to drive the initial solidification. 


\subsection{SPECIFIC ICING MODELS}

The simulation of ice accretion on aerofoils starts with a computation of the airflow, followed by successive computation of water droplet trajectories, collection efficiency and finally the thermodynamic balance to determine the ice profile. The airflow computation could be from a Navier-Stokes solver, or a coupled inviscid flow solver with an integral boundary layer correction. Although the inviscid solver could be based on solution to the Euler equations, it is more likely to tackle potential flow using either panel methods ([30], [31]) or finite-difference methods [32]. The water droplet trajectories and collection efficiency calculations are detailed in [3] while the ice-prediction typically follows from the energy-balance approach of [34], involving kinetic heating, convective cooling, latent heat of freezing and several sensible heat terms. Finally, the roughness which develops on ice-covered surfaces controls the boundary-layer flow which, in turn, affects droplet efficiency and heat transfer. Thus the modelling of surface roughness, as described by [35], [31], is of much importance. Further [35] provide time-dependent results to compare both with experiments and our own analysis.

Very much related to the above are the theoretical investigations of [36], [37], [38], [39], and [40]. A small aspect ratio and reduced Reynolds number allow the fluid flow to be modelled by lubrication theory. Analytical solutions for the velocity components yield a fourth-order nonlinear partial differential equation for the free surface which in turn reduces to a very simple result for rime icing (no liquid layer). In essence this is similar to the triple-deck approach of [41] although there the resulting nonlinear boundary layer system requires numerical evaluation. Of more relevance to our study is the numerical and experimental investigation by [42] into the oblique impact and freezing of a supercooled large droplet (SLD) onto a thin layer of water. A series of Navier-Stokes simulations show the formation and break-up of ejecta jets seen in experiments. To simulate ice formation, a thin array of triangular seed crystals, at the freezing temperature, is initially set at the bottom of the water layer. The isolated crystals first grow into each other and then into the supercooled droplet water. The ice layer modifies the flow of the droplet and layer water and so affects the form of splash.

The present theoretical study is concerned primarily with events near the instant of impact. The seminal work on such droplet impacts and the related issue of water-entry problems was carried out by [43]. [44] identified the importance of compressibility and the significance of the shock wave formed upon impact. They argued that for high-speed impacts the time at which the shock wave overtakes the contact point is the start of the spreading stage (see [45]). For lower-speed impacts this description of the lamella origin is rejected by [46] who identify the importance of ambient air pressure, arguing that initially the droplet spreads on a thin sheet of air. Above a critical 
impact velocity, they suggest the lamella is ejected before surface contact which, subsequently, deflects the lamella upwards, causing the corona splash described above. Other analytical work includes [47], [48], [49], [50], [51], and [52] who studied water entry problems at low deadrise angles and other impact problems. Droplet impacts into water layers have been presented by [53], [54], while [55], [56] also derived many of the interesting air-cushioning results found independently in [46]. Recent work includes impacts on rough surfaces by [57] and skimming and rebounding by [58].

It is also worthwhile to consider another classical heat diffusion process, namely the unconstrained planar solidification of a pure substance from an undercooled melt initially at a temperature $T_{\infty}^{*}$. If $T_{f}^{*}$ is the equilibrium fusion temperature, then the process is parameterised by the Stefan number $S t$, a dimensionless ratio of the temperature difference $\Delta T^{*}=T_{f}^{*}-T_{\infty}^{*}>0$ and the latent heat at the fusion temperature. The classical Stefan formulation, see [59] and [60], of a square-root diffusion controlled growth with a sharp front held at the equilibrium fusion temperature $T_{f}^{*}$ breaks down for rapid solidification, there being no solution for $S t \geq 1$ [61]. This is due to a reduction in the temperature from that of equilibrium at the interface, or so-called kinetic undercooling. One way to resolve this is the phase-field approach, see [62], which allows for a finite thickness of the front. A second approach is to retain a sharp front but incorporate the effects of interfacial kinetics by relating the drop in fusion temperature from equilibrium to the rate of solidification. The change in the solidification temperature is accompanied by a corresponding change in the latent heat, which is a function of the interfacial temperature, implying entropy production at the interface when there is a difference in the specific heat between the two phases. The simplest case, a linear relationship, is appropriate to molecularly rough or unfacetted interfaces. There have been many studies in this area including notably [63], [64], [65], [66], [67], [68] and [69]. Not all of the above consider the effects of density and specific heat jumps upon change of phase or even allow for the corresponding change in the latent heat. Some consider only the single phase process in isolation from the temperature distribution of the substrate.

\subsection{THE PRESENT STUDY}

The prime focus here is on ice growth, surface roughness and relevant viscous effects, with the surface roughness representing pre-existing icing. For simplicity in our study we shall consider only rough surfaces that are symmetrical about the single initial point of contact. Compressible and acoustic effects are neglected, partly again for simplicity but also in line with the [70] recommendations. In fact with the application to aircraft icing and the main physical effects foremost in mind the influences of air need not be accommodated in a first model and the droplet is considered to move through a void. In addition 
for convenience we shall fix the temperature variation within the accreted ice. The Reynolds and Weber numbers are assumed large, so in particular as a first approximation we ignore the effects of surface tension. It might appear that we can also treat the droplet as inviscid. However viscous effects cannot be fully ignored if the effects of the water droplet freezing are to be considered. The interplay between the droplet and the growing ice surface adds to the apparent novelty of the present work. Moreover, the model we use deals with small time scales in early build-up of ice for a single droplet and then addresses by extrapolation many droplets by means of their frozen roughness effect.

The plan of the paper is as follows. In $\$ 2$ the interaction between ice accretion and the fluid flow is explored for a representative physical configuration. Then in $\$ 3$ scaling arguments focus attention on the regime which covers aircraft icing where the relative size of the ice accretion that first affects significantly the non-ice behaviour is identified. Viscous effects and multi-structure come rather to the fore here. Next $\S 4$ examines the viscous and inviscid outer response, the primary area of concern for determining ice shapes. Wet-side and dry-side layers are examined. (We note the analysis also covers as a special case the viscous effects in an isothermal impact without phase change as well as describing differences between impact with and without freezing.) Following this $\S 5$ presents numerical findings for the resulting integral system while $\$ 6$ summarises briefly the innermost walllayer responses. Finally in $\$ 7$ comparisons are made with experiments to check on the present predicted orders of magnitude primarily, followed by closing comments.

\section{The interplay of accretion and flow}

For definiteness let us consider a single two-dimensional supercooled cylindrical water droplet of radius $R_{D}^{*}$ and temperature $T_{D}^{*}<T_{f}^{*}$, where $T_{f}^{*}$ is the equilibrium solidification temperature and the $z^{*}$-axis is the generator of the infinite cylinder. The cylinder moves towards the cylinder in the negative $y^{*}-$ direction, subject only to a gravitational acceleration $g^{*}$, through a void at a pressure $p_{\infty}^{*}$ with a constant velocity $U_{D}^{*}$, such that at time $t^{*}=0$ it impacts onto a rough surface at the single point $x^{*}=y^{*}=0$. The rough surface itself lies on top of a fixed planar substrate at $y^{*}=-r_{0}^{*}$. The appearance of splash jets is expected as the drop spreads but additionally there is the accretion of ice over the wetted part of the rough surface, with an unknown water-ice interface at a temperature $T_{c}^{*} \leq T_{f}^{*}$, due to kinetic undercooling.

Throughout an asterisk denotes a dimensional quantity and its absence denotes a dimensionless quantity. Thus the co-ordinates $(x, y)$, the time $t$ and the corresponding velocity components $(u, v)$ are made dimensionless 
with respect to droplet radius $R_{D}^{*}$, representative droplet travel time $R_{D}^{*} / U_{D}^{*}$ and typical speed $U_{D}^{*}$ respectively. We also define $\rho_{L}^{*}, \mu_{L}^{*}, \sigma_{L}^{*}, K_{L}^{*} c_{p, L}^{*}$ and $\alpha_{L}^{*}=K_{L}^{*} /\left(\rho_{L}^{*} c_{p, L}^{*}\right)$ in turn to be the density, viscosity, surface tension, thermal conductivity, specific heat and thermal diffusivity of the fluid, all assumed constant, and $p_{\infty}^{*}+\rho_{L}^{*} U_{D}^{* 2} p$ and $T_{D}^{*}+\left(T_{f}^{*}-T_{D}^{*}\right) T$ to be the pressure and temperature of the fluid. In non-dimensional terms the droplet is a unit circle of supercooled fluid at zero temperature descending with unit speed with an equilibrium fusion temperature of unity. Finally if $T_{D}^{*}+\left(T_{f}^{*}-T_{D}^{*}\right) T_{I}$ is the temperature of the accreted ice, then we define $\rho_{i c e}^{*}, K_{i c e}^{*}, c_{p, i c e}$ and $\alpha_{i c e}^{*}$ to be the corresponding (constant) values of the quantities for the ice phase. Consequently the Reynolds number $R e$, Froude number $F r$ and Weber number $W e$ are defined according to

$$
R e=\frac{\rho_{L}^{*} U_{D}^{*} R_{D}^{*}}{\mu_{L}^{*}}, \quad F r=\frac{U_{D}^{* 2}}{g^{*} R_{D}^{*}}, \quad W e=\frac{\rho_{L}^{*} U_{D}^{* 2} R_{D}^{*}}{\sigma_{L}^{*}} . \quad(2.1 a-c)
$$

The gravity and capillary effects turn out to be negligible in the current real context but are included for completeness at this stage. In addition the Prandtl number $P r$, Stefan number $S t$ and the kinetic underheating parameter $\beta$ are defined by

$$
\operatorname{Pr}=\frac{\mu_{L}^{*} c_{p, L}^{*}}{K_{L}^{*}}, \quad S t=\frac{c_{p, L}^{*} \Delta T^{*}}{L_{f}^{*}}, \quad \beta=\frac{U_{D}^{*}}{G \Delta T^{*}}, \quad(2.1 d-f)
$$

where $\Delta T^{*}=T_{f}^{*}-T_{D}^{*}>0$ is the difference between the drop temperature and the equilibrium fusion temperature $T_{f}^{*}$, while $L_{f}^{*}$ is the latent heat of fusion at $T_{f}^{*}$ and the constant $G$ is the kinetic coefficient characterising the frequency of molecular bonding. Finally, following [71], we have the capillary cooling parameter, $\Gamma$, defined by

$$
\Gamma=\frac{T_{f}^{*}}{\Delta T^{*}} \frac{\sigma_{L}^{*}}{\rho_{L}^{*} R_{D}^{*} L_{f}^{*}}=\frac{T_{f}^{*}}{\Delta T^{*}} \frac{S t}{W e} E c \quad \text { where } \quad E c=\frac{U_{D}^{* 2}}{c_{p, L}^{*} \Delta T^{*}}
$$

is the Eckert number. Numerical values for the parameters in (2.1a-g) are discussed at the end of this section.

We define $y=h^{ \pm}(x, t)$ for $|x|>d(t)$ as depicted in figure 1 to be the unknown free surface shape, where $x= \pm d(t)$ are the turnover points with $h^{+}( \pm d(t), t)=h^{-}( \pm d(t), t)$. The surface roughness is given by $y=r(x)$ such that $r(0)=0$ with $r(x) \geq-r_{0}$ together with the assumption of symmetry about $x=0, r(-x)=r(x)$, requiring only the solution in $x>0$ to be considered. That is we assume the droplet impacts at either a local minimum or maximum of the surface roughness, which may have a significant limitation as far as the real application is concerned. The so-called roughness is taken to be reasonably smooth in mathematical terms. Finally here 


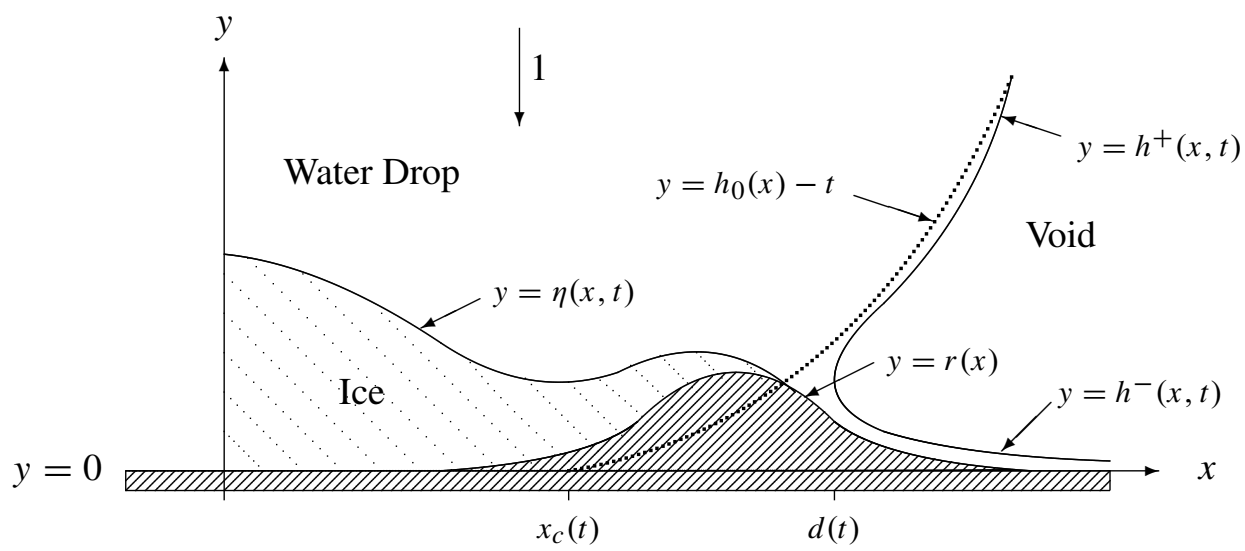

Figure 1. Schematic representation of flow on a rough surface of height $r$, with unknown interfaces at heights $\eta$ (ice), $h^{ \pm}$(water droplet)

$y=\eta(x, t)$ is the water-ice surface which is at a temperature $T_{c} \leq 1$. The situation (for $r>0)$ is sketched in figure 1 for early times. If $\mathbf{u}=(u, v, 0)$ is the fluid velocity vector then within the drop the governing equations are

$$
\begin{gathered}
\nabla \cdot \mathbf{u}=0, \\
{\left[\frac{\partial}{\partial t}+(\mathbf{u} \cdot \nabla)\right] \mathbf{u}=-\nabla p+\frac{1}{F r}\left(\sin \alpha_{0},-\cos \alpha_{0}, 0\right)+\frac{1}{\operatorname{Re}} \nabla^{2} \mathbf{u},} \\
{\left[\frac{\partial}{\partial t}+(\mathbf{u} \cdot \nabla)\right] T=\frac{1}{\operatorname{Pr} R e} \nabla^{2} T .}
\end{gathered}
$$

The direction of the gravity effect depends on the constant angle $\alpha_{0}$. Next, the boundary conditions account for continuity of velocity, temperature, fluxes and stresses effectively at the given solid base, at the unknown ice-water interface and at the unknown water-void free surface, supplemented by the influences of capillary contributions and latent heat. At the ice-water interface $y=\eta(x, t)$ continuity of tangential flow, conservation of mass, and continuity of temperature need to be applied. In addition there is a need to balance the heat flowing into/out of the interface and the heat lost/gained by solidification. Consequently

$$
\begin{gathered}
u+v \frac{\partial \eta}{\partial x}=0, \quad v-u \frac{\partial \eta}{\partial x}=(1-\rho) \frac{\partial \eta}{\partial t}, \\
T=T_{I}=T_{c} \equiv 1+\frac{1}{\sqrt{1+\eta_{x}^{2}}}\left(\frac{\Gamma}{\rho\left(1+\eta_{x}^{2}\right)} \frac{\partial^{2} \eta}{\partial x^{2}}-\beta \frac{\partial \eta}{\partial t}\right), \\
{\left[\frac{\rho}{S t}-\left(1-\rho c_{p}\right)\left(1-T_{c}\right)\right] \frac{\partial \eta}{\partial t}}
\end{gathered}
$$




$$
=\frac{1}{\operatorname{PrRe}}\left[K\left(\frac{\partial T_{I}}{\partial y}-\frac{\partial \eta}{\partial x} \frac{\partial T_{I}}{\partial x}\right)-\left(\frac{\partial T}{\partial y}-\frac{\partial \eta}{\partial x} \frac{\partial T}{\partial x}\right)\right] .
$$

See [28], [36], [59], [71]. Here $\rho=\rho_{i c e}^{*} / \rho_{L}^{*}<1, c_{p}=c_{p, i c e}^{*} / c_{p, L}^{*}$ and $K=K_{i c e}^{*} / K_{L}^{*}$ are the corresponding ratios for the parameters within the two phases. In fact, due to the relative thinness of the accreted ice with or without roughness, we can effectively set $u=0$ at the ice-water interface.

In $(2.3 \mathrm{c})$ kinetic and capillary undercooling are modelled using a linear Gibbs-Thompson relationship between the departure of the fusion temperature from equilibrium and both the solidification rate and curvature of the ice-water interface. In (2.3d) we have assumed a similar linear departure for the latent heat from its equilibrium value. For $\beta=\Gamma=0$ we recover the classical Stefan formulation for the solidification of a pure substance from an undercooled melt, by a sharp front held at the equilibrium solidification temperature $T_{f}^{*}$ and latent heat $L_{f}^{*}$. The simplified case $\rho=c_{p}=1$, corresponding to no change in density upon solidification, leads to the no-slip condition at the interface.

At the void-water interface $y=h^{ \pm}(x, t)$ the kinematic condition, continuity of temperature and a linear momentum balance between the stress and surface force (see [71]) apply,

$$
v=\frac{\partial h^{ \pm}}{\partial t}+u \frac{\partial h^{ \pm}}{\partial x}, \quad T=0, \quad \hat{\mathbf{n}} \cdot \mathbf{T} \cdot \hat{\mathbf{n}}=\frac{\kappa}{W e},
$$

where $\mathbf{T}=-p \mathbf{I}+R e^{-1} \mathbf{S}$ is the Newtonian stress tensor with $\mathbf{S}=(\nabla \mathbf{u})+$ $(\nabla \mathbf{u})^{T}$. Here $\hat{\mathbf{n}}$ is the outward normal to the interface, directed from the drop into the void, and $\kappa=-\nabla \cdot \hat{\mathbf{n}}=\mp h_{x x}^{ \pm} /\left(1+h_{x}^{ \pm 2}\right)^{3 / 2}$ is the dimensionless curvature. For an inviscid fluid this reduces to the usual Laplace relation for the jump in the pressure which in turn reduces to the Bernoulli condition $p=0$, or continuity of pressure, upon neglect of surface tension.

The relevant initial conditions at time $t=0$ are

$$
\begin{aligned}
& u=0, \quad v=-1, \quad T=0 \quad \text { for } x^{2}+(y-1)^{2}<1, \quad(2.4 a-c) \\
& h^{+}(x, 0)=h_{0}(x), \quad \eta(x, 0)=r(x) \geq 0, \quad d(0)=h^{-}(x, 0)=0,
\end{aligned}
$$

which together with $r(x) \leq h_{0}(x)=1-\sqrt{1-x^{2}}$ ensure that the circular droplet initially impacts at a single point in the absence of ice accretion. Both the icing and splash jet are localised to the vicinity of the droplet.

Finally, within the ice region, there is only conduction of heat subject to continuity of temperature at the interface and zero heat flux at the given rough solid surface at $y=r(x)$. As discussed below, the zero heat flux condition allows us to assume that the temperature distribution $T_{I} \approx T_{c}$, which greatly simplifies (2.3d). Gaining insight into the whole system (2.2a)-(2.4f) represents the central task below. 
The main application in mind is aircraft icing for which typical speeds are of the order of $V^{*}\left(=U_{D}^{*}\right)=150 \mathrm{~ms}^{-1}$ and the typical droplet diameters range from $D^{*}\left(=2 R_{D}^{*}\right)=40 \mu \mathrm{m}$ to $400 \mu \mathrm{m}$, [3]: see also $\$ 7$ below. Thus the Reynolds number $R e$ based on droplet radius is around $10^{4}$ which is large and the droplet is treated in most parts as inviscid as a first model, subject to boundary-layer effects. The Weber number $W e \approx 10^{5}$ is also large and so surface tension effects can be excluded as a first approximation. The Froude number is large (typically $10^{7}$ or so) and the gravity effect is therefore negligible in the setting of this study. The Prandtl number is about 7-13 for water and the capillary underheating parameter $\Gamma$ is small (typically $10^{-5}-10^{-6}$ ). We shall assume that the Stefan number and the kinetic underheating parameter are order unity. In fact $S t$ is wide ranging, from $10^{-2}$ to 10 say, and depends on the temperature differences, whilst $\beta$ is also very temperature-dependent and speed-dependent.

\section{Scaling arguments}

We wish to extend the inviscid small-time analysis of [53] and [49] to incorporate both small surface roughness (earlier freezings), as described by [57], and ice accretion. In the absence of solidification and surface roughness we would expect $d \propto \sqrt{t}$ for $0<t \ll 1$, a result one can obtain purely from geometric considerations. With solidification and roughness present the same result can only apply in the sense of an order of magnitude as will be seen; the importance of the outer regions studied in previous papers will also be seen here but coupled now with inner regions because of the ice accretion.

\subsection{ICE ACCRETION AND DIFFUSIVE EFFECTS}

To understand the effect of kinetic undercooling in isolation, and based on a one-dimensional thermal investigation $(r=0)$ for $\beta=O(1), \Gamma=0$ and $R e \gg 1$ by [64] amongst others, for $\rho=c=1$, we might expect two time intervals to apply. They come from comparing the $y$-scale due to temporal growth in $(2.2 b)$ with the beta effect in $(2.3 \mathrm{c})$. Thus for relatively early times the actively freezing layer and the frozen layer underneath are given respectively by

$$
y-\eta \propto(\operatorname{RePr})^{-\frac{1}{2}} \sqrt{t}, \quad \eta \propto \beta^{-1} t, \quad \text { for } \quad 0<t \ll \beta^{2} \operatorname{Re}^{-1},(3.1 a, b)
$$

where $T_{I} \approx T_{c}=O\left[(\operatorname{Re} \operatorname{Pr})^{\frac{1}{2}} \sqrt{t} / \beta\right] \ll 1$, or $T_{c}^{*} \approx T_{D}^{*}$. For later times $\beta^{2} R e^{-1} \ll t \ll 1$ there is a dual form

$$
y-\eta=O\left[(\operatorname{RePr})^{-1} \beta\right], \quad \eta=O\left(\beta^{-1} t\right), \quad \text { for } \quad S t>1, \quad(3.1 c, d)
$$




$$
y-\eta \sim \eta=O\left[(\operatorname{RePr})^{-\frac{1}{2}} \sqrt{t}\right] \text { for } \quad S t<1 .
$$

In both cases the ice temperature $T_{I} \approx T_{c}=O(1)$, and indeed $T_{c}-1$ is exponentially small for $S t<1$, or $T_{c}^{*} \approx T_{f}^{*}$. The role of the Peclet number $\operatorname{RePr}$, independent of viscosity, is clear. The specific result $\eta \sim R e^{-\frac{1}{2}} \Lambda \sqrt{t}$ for $S t<1$, where the order-unity constant $\Lambda$ satisfies the eigen-relation [71], [61]

$$
S t=\frac{1}{2} \sqrt{\pi \operatorname{Pr}} \rho \Lambda \operatorname{erfc}\left[\frac{1}{2} \sqrt{\operatorname{Pr}} \rho \Lambda\right] \mathrm{e}^{\frac{1}{4} \operatorname{Pr}(\rho \Lambda)^{2}},
$$

matches to the classical $(\beta=0)$ similarity solution of the one-dimensional Stefan problem for solidification of an undercooled melt with a square-root accretion growth. In contrast, the analysis of the type given by [64] suggests $\eta \sim \gamma t$ for $S t>1$ which is a range of Stefan number of some interest in the present study. This faster linear solidification growth where the constant $\gamma$ is given by

$$
\gamma=\frac{\rho}{\beta\left[\rho-\left(1-\rho c_{p}\right)\right]}\left(1-\frac{1}{S t}\right)>0
$$

reflects the fact, see [61], that the classical one-dimensional Stefan problem actually has no such similarity solution for $S t \geq 1$. One might argue that the small time scale $t=O\left(R e^{-1}\right)$ models the rapid recalescence stage of freezing, identified by [6] amongst others, in which the released latent heat of solidification is used to raise the interfacial droplet temperature from $T_{D}^{*}$ to the vicinity of the equilibrium freezing temperature $T_{f}^{*}$.

As well as kinetic undercooling being important mathematically for both the removal of singularities and non-existence of solutions found in the classical Stefan representation of solidification and melting processes, the algebraic and exponential forms for the dependence of the undercooling on the interface growth rate match the experimental data for a wide range of applications, namely crystal growth, cellular alloy solidification, solid fuel combustion, penetration of solvents into glassy polymers and oxidation of silicon. See data in [5], [31], [7], [6] for example. In the configuration of current interest a value of the Stefan number $S t$ greater than unity corresponds to the incident droplet being severely undercooled.

\subsection{The MULTi-STRUCTURE}

Motivation and a starting point indicating roughness effects are provided by the inviscid structure of [57] for a small scaled time $t \sim \varepsilon^{2}$ where the constant $\varepsilon$ is small and positive. As indicated by figure 2 in which $\delta$ is $\varepsilon^{-2} R e^{-\frac{1}{2}}$ and is small, there is a complicated interactive structure now in the presence of ice formation. First there is an outer region I $a$ close to the point of impact, when $x, y \sim \varepsilon$. This is a region of potential isothermal flow in which both 
velocities $u, v$ are mostly $O(1)$, the temperature $T \equiv 0$, and the pressure response $p \sim \varepsilon^{-1}$ is large to balance the large acceleration. In addition, the free surface shape has $h^{+} \sim \varepsilon^{2}, h^{-} \sim \varepsilon^{3}$ and surface roughness has $r \sim \varepsilon^{2}$, implying there is also a thin layer $I b$ where $y \sim \varepsilon^{2}$. The scaling on roughness is that which, as the roughness height is gradually increased, first affects the evolution nonlinearly; this roughness height or the typical icing height can also be used to specify the small parameter $\varepsilon$, and similar considerations hold for the roughness length. To fully consider the solution we note there are inner potential regimes $I I a$ and $I I I a$, centred on the unknown moving point of contact $x=d$ where $x-d, y \sim \varepsilon^{2}$ and $\varepsilon^{3}$, respectively, that enable the flow to accommodate the impact. We also note that since $R e \gg 1$ the problem in region $I$ is inviscid as indicated already but for the problem to remain inviscid in regions $I I$ and $I I I$ the requirements $\varepsilon \gg R e^{-1}$ and $\varepsilon \gg R e^{-\frac{1}{2}}$ apply. Finally there is an additional so-called splash-jet region $I c^{+}$. This is a thin layer of fast moving fluid downstream of the contact point where $x \sim \varepsilon$, $y \sim \varepsilon^{3}$ for which $u$ is $O\left(\varepsilon^{-2}\right)$ and where the restriction $\varepsilon \gg R e^{-\frac{1}{4}}$ ensures the flow remains as if inviscid. This also tends to suggest that the later time scale $(3.1 \mathrm{c}, \mathrm{d})$ rather than $(3.1 \mathrm{a}, \mathrm{b})$ is relevant here.

Ice accretion or solidification, at least for Prandtl number $\operatorname{Pr}$ of $O(1)$, occurs over viscous-like scales and therefore for the purpose of understanding the accretion response we have to examine a thin inner viscous layer satisfying the no-slip condition and the kinematic and temperature conditions $(2.3 \mathrm{~b}, \mathrm{c})$ at the ice-water interface. In such a layer the vertical coordinate $\zeta \equiv y-\eta(\bar{x}, \bar{t}) \ll \varepsilon^{2}$ and so because of the vertical shift a Prandtl-like transformation is adopted,

$$
\begin{gathered}
u=u_{L}(\bar{x}, \zeta, \bar{t}), \quad p=p_{L}(\bar{x}, \bar{t}), \quad T=T_{L}(\bar{x}, \zeta, \bar{t}), \\
v=\frac{1}{\varepsilon^{2}}(1-\rho) \frac{\partial \eta}{\partial \bar{t}}+\frac{1}{\varepsilon} \frac{\partial \eta}{\partial \bar{x}} u_{L}(\bar{x}, \zeta, \bar{t})+v_{L}(\bar{x}, \zeta, \bar{t}),
\end{gathered}
$$

where $x=\varepsilon \bar{x}, t=\varepsilon^{2} \bar{t}$. Here the case $\rho=1$ would correspond to no volume change upon solidification and no-slip at the ice-water interface. Finally, for precision, $r=\varepsilon^{2} \bar{r}(\bar{x})$ and $d=\varepsilon \bar{d}(\bar{t})$ define the assumed known surface roughness height and the unknown contact point respectively.

\subsection{ICE ACCRETION IN DETAIL, UPSTREAM AND DOWNSTREAM}

The conditions at the water-ice interface $(2.3 \mathrm{c}, \mathrm{d})$ imply that for $T_{L} \sim T_{c}$ of $O(1)$ with $S t$ of order unity the scaling $\eta \sim \varepsilon^{2} / \beta$ allows for kinetic undercooling. Continuity implies $v_{L} \sim \zeta u_{L} / \varepsilon$ whilst retaining the unsteadypressure balance of the outer region $I a$ leads to $p_{L} \sim \varepsilon^{-1} u_{L}$. A fully unsteady pressure-driven viscous sublayer $I v$ then gives rise to the scalings

$$
\frac{u_{L}}{\varepsilon^{2}} \sim \frac{\eta}{\varepsilon^{2} \zeta} u_{L} \sim \frac{p_{L}}{\varepsilon} \sim \frac{u_{L}}{\operatorname{Re} \zeta^{2}} .
$$




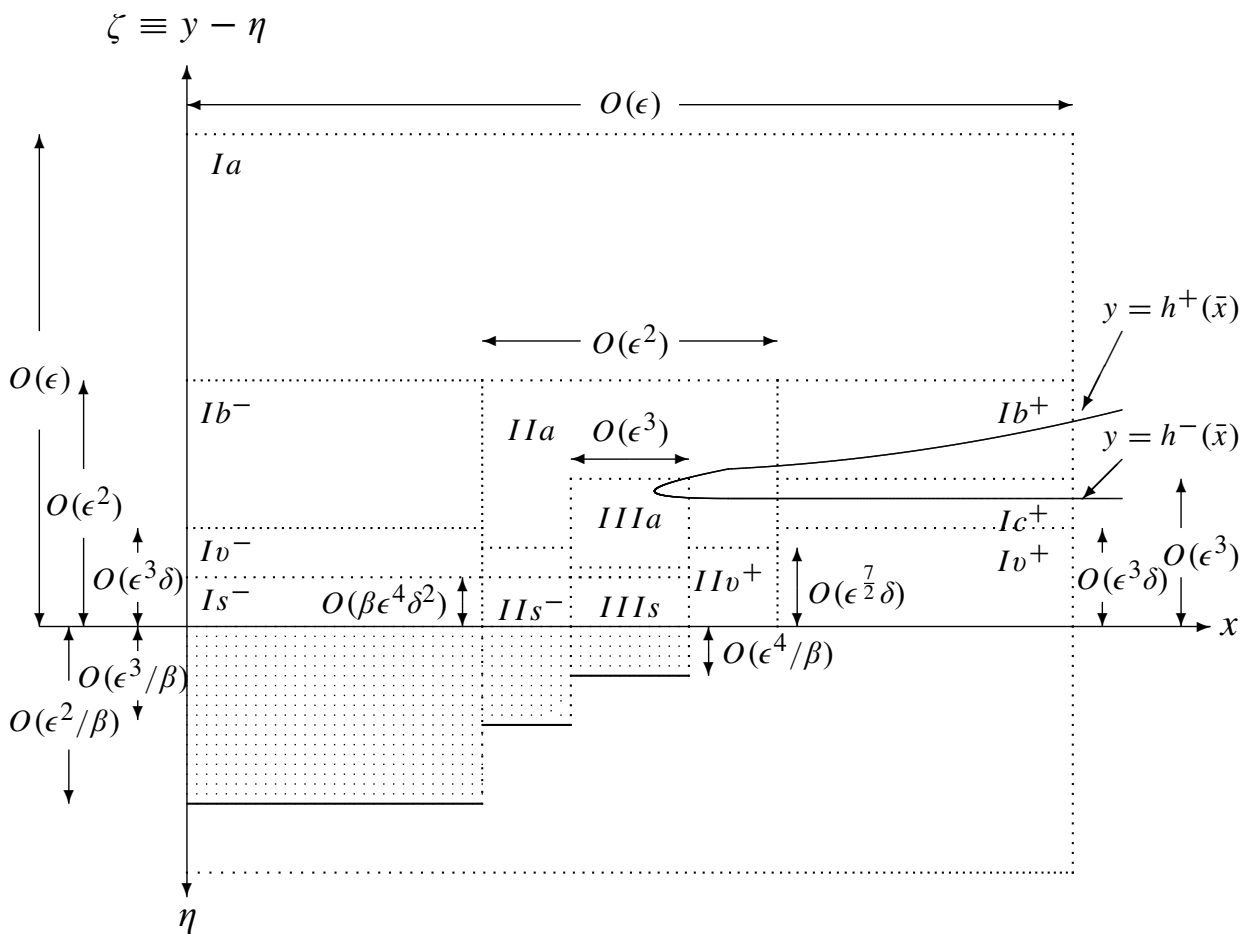

Figure 2. The relatively fast solidification structure $(S t>1)$, where the subscript $s$ refers to linear quasi-steady inner regions lying within the viscous (v) regions of a slower solidification structure on the wet-side of the contact point; superscripts \pm refer to dry (+) and wet (-) sides.

This in turn gives rise to the orderings $\zeta \sim \eta \sim \varepsilon^{3} \delta, v_{L} \sim \zeta u_{L} / \varepsilon=\varepsilon^{2} \delta u_{L}$ where we recall $\delta=\varepsilon^{-2} R e^{-\frac{1}{2}} \ll 1$, which matches with the limit $(3.1 c, d)$ for St $<1$. The dynamics are linear provided the velocity $u_{L} \ll \varepsilon^{-1}$, and indeed matching to $I a$ implies $u_{L}=O(1)$. We note then that $\eta \sim \varepsilon^{3} \delta \ll \varepsilon^{2} / \beta$, which corresponds to the classical Stefan problem (no undercooling) with temperature $T_{c} \equiv 1$. An analysis of viscous sublayers $I I v$ and $I I I v$ similar to that for inner regions II $a$ and II I $a$ respectively, where $x-\varepsilon \bar{d}=O\left(\varepsilon^{n+1}\right)$ for $n=1,2$, then yields thicknesses $\zeta \sim \eta \sim \varepsilon^{(6+n) / 2} \delta$ and velocities $v_{L} \sim$ $\varepsilon^{(4-n) / 2} \delta u_{L}, u_{L}=O\left(\varepsilon^{-n / 2}\right)$. The asymptotic structure is as depicted in figure 2 , without the regions denoted ' $s$ ', and the accreted ice is indicated by the shaded regions.

Alternatively if kinetic undercooling takes place within a sublayer $I \mathrm{~s}^{-}$, suggesting a quasi-steady linear viscous flow independent of the pressure, then

$$
\frac{\eta}{\varepsilon^{2} \zeta} u_{L} \sim \frac{u_{L}}{\operatorname{Re} \zeta^{2}}, \quad \eta \sim \frac{\varepsilon^{2}}{\beta}
$$


This, in turn, implies the orderings $\zeta \sim \beta \varepsilon^{4} \delta^{2}, v_{L} \sim \zeta u_{L} / \varepsilon=\beta \varepsilon^{3} \delta^{2} u_{L}$ with $\beta R e^{-\frac{1}{2}}=\beta \varepsilon^{2} \delta \ll \varepsilon \ll 1$, which matches with the limit (3.1c,d) for $S t>1$. Once again a similar analysis of viscous sublayers IIs and IIIs leads to $\zeta \sim \beta \varepsilon^{4} \delta^{2}, \eta \sim \varepsilon^{2+n} / \beta, v_{L} \sim \beta \varepsilon^{3-n} \delta^{2} u_{L}, u_{L}=O\left(\varepsilon^{-n / 2}\right)$ and a fastersolidification structure as depicted in figure 2 . Here the quasi-steady sublayers denoted by the subscript $s$ are much thinner than their slower solidification counterparts which are denoted in figure 2 by the subscript $v$.

Given that there appear to be two sets of asymptotic structures (figure 2 ) depending on whether $S t \gtrless 1$ we observe that at the limit of the range of validity of the above analysis, namely the earliest time when $\delta=O(1)$ $\left(\varepsilon \sim R e^{-\frac{1}{4}}\right)$, the different inner sublayers IIIv/s of figure 2 are identical if $\beta$ is of $O(1)$ whilst the splash jet is viscous. This implied fundamental inner interaction in which $\varepsilon \sim R e^{-\frac{1}{4}}$ consists of the quasi-steady nonlinear classical boundary-layer, subject to a prescribed stagnation-type slip-velocity and an upstream-moving wall with the complications of ice-growth equivalent to blowing or suction at the rigid wall. This governs the flow for all $S t$ and incorporates both kinetic and capillary underheating provided the parameter $\Gamma$ is $O\left(R e^{-\frac{1}{2}}\right)$. It is at the heart of all subsequent small- $\delta$ innermost sublayers, notably $I I I v^{+} / I I I s$ in figure 2 except that subsequently, for such a sized capillary parameter, underheating is negligible.

The flow solution for the above fundamental problem matches asymptotically to both viscous sublayers $I v^{-} / I s^{-}$upstream of the moving contact point whereas an interesting issue is encountered in matching the 'downstream' form to the viscous sublayers $I v^{+}$beneath the splash-jet, on the 'dry' side of the contact point. Orders of magnitude suggest first that the downstream asymptotic problem might reduce to one of solving the Blasius equation for a moving wall, namely

$$
f_{B}^{\prime \prime \prime}+\frac{1}{2} f_{B} f_{B}^{\prime \prime}=0, \quad f_{B}(0)=-C, \quad f_{B}^{\prime}(0)=-1, \quad f_{B}^{\prime}(\infty)=1 .
$$

Here $C=0$ corresponds to the absence of ice accretion, whilst $C>0$ corresponds to a seemingly non-physical square-root growth in the ice thickness into the splash jet. Unfortunately [72], who considered the case $f_{B}^{\prime}(0)=-\lambda$, showed that solutions exist only for $\lambda<\lambda_{c} \approx 0.3541$. Any hope that solidification might alleviate this problem is dashed by the several authors, most recently [73], who considered the case $f_{B}(0)=-C, f_{B}^{\prime}(0)=-\lambda$. They also found solutions for $\lambda<\lambda_{c}$ but additionally that increasing $C$ caused $\lambda_{c} \rightarrow 0^{+}$. Instead we must use the evidence that a solid wall moving upstream relative to the outer stream tends to produce flow separation and accompanying re-scaling either of relatively confined form $[74,75]$ or of breakaway or bursting form [76] both of which are highly unstable and lead readily to quite abrupt transition to turbulence $[77,78,79,80]$. Thus the viscous sublayer flow $I v^{+}$beneath the splash jet seems almost certain to be highly separated, 


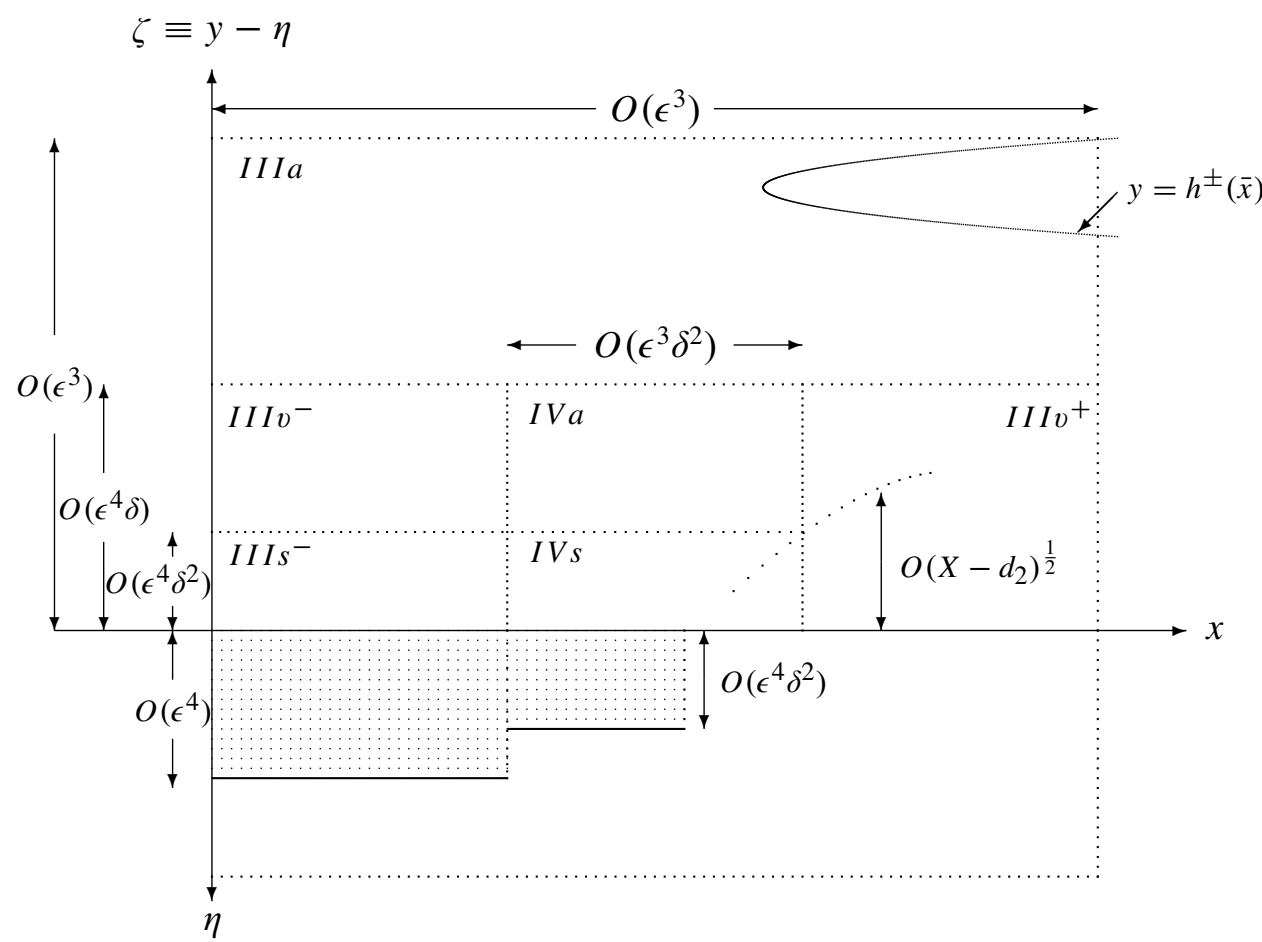

Figure 3. The innermost asymptotic structure $(S t>1)$

transitional and turbulent in practice. The effect of the moving contact point is to sweep away the turbulent flow to leave behind the laminar structure of regions $I v^{-} / I s^{-}$; yet the identification of such flow however limited it is represents an intriguing property.

The above property in the 'downstream' asymptote is the reason why in the above figures no attempt is made to obtain precise scalings for the ice thickness on the 'dry' side of the contact point. Another intriguing issue here is that the so-called dry side is in fact over-ridden by a comparatively thin splash jet of water. It seems reasonable though on physical grounds for us to expect there to be considerably more ice on the wetted side $x<d(t)$ of the contact point, where the majority of the liquid is available for removal of heat by latent heat of conduction, than on the dry side $x>d(t)$. With this in mind let us reasonably suggest that $\eta \ll O\left(\varepsilon^{3} \delta\right)$ in region $I$ downstream (the dry side) of the contact point. It actually seems not entirely impossible that the solidification is comparable on the two sides of the contact point but the present approach works with a significant contrast between the wet-side and dry-side features of accretion.

Closer in, very near the turnover position, figure 3 depicts the configuration. For the case of $S t>1$, the inner viscous region IIIS is linear. 
Supposing that at some point say $X=\bar{d}$ the ice thickness $\eta=\varepsilon^{4} \tilde{\eta}$ vanishes, we would expect nonlinearity to re-assert itself and eventually the dynamics of region $I I I v^{+}$to govern. An inertial-viscous balance

$$
\left(u-\frac{1}{\varepsilon} \frac{\mathrm{d} \bar{d}_{0}}{\mathrm{~d} t}\right) \frac{\partial u}{\partial x} \sim \frac{\partial p}{\partial x} \sim \frac{1}{\operatorname{Re}} \frac{\partial^{2} u}{\partial \zeta^{2}}
$$

implies $\zeta^{2} \sim \varepsilon^{5} \delta^{2} x$ since $u=O\left(\varepsilon^{-1}\right), p=O\left(\varepsilon^{-2}\right)$ in region IIIs. That is $\hat{Y} \sim\left(X-\bar{d}_{2}\right)^{\frac{1}{2}}$ where $\zeta \equiv y-\eta=O\left(\varepsilon^{4} \delta\right)$ and $x-d=O\left(\varepsilon^{3}\right)$ are the scales for region IIIV. One possibility would require the introduction of an innermost viscous layer $I V s$ with $\zeta=O\left(\varepsilon^{4} \delta^{2}\right)$, the same thickness as $I I I s$, when $x-d=O\left(\varepsilon^{3} \delta^{2}\right)$. This structure is depicted in figure 3. The downstream asymptotic form of this innermost region involves a square root growth which provides the starting form for the solution in $I I I v^{+}$. This smoothing of the solution for $S t>1$ at the point where the ice accretion disappears does not affect the issues with the downstream asymptotic form alluded to above.

\section{The outer response and the ice shapes}

The prediction of the ice shape produced by the impact is significant since it enables comparisons to be made more readily with experiments in principle. The prediction relies mostly on the interactive behaviour in the outermost regions of the multi-structure although interesting features emerge also in the inner regions around the ice.

Here and below the scalings from the previous section lead to the waterflow/ice-shape interactions which involve quantities that are fully determined in $\S 5$. As inferred above, we take $t=\varepsilon^{2} \bar{t}$ and in this interval set $x=\varepsilon \bar{x}$ with $\bar{x}=O(1)$ with an assumed turnover (contact) point at $\bar{x}=\bar{d}_{0}(\bar{t})$. In addition, for $\bar{x}>\bar{d}_{0}(\bar{t})$ the free surface is described by $h^{+}=\varepsilon^{2} \bar{h}^{+}(\bar{x}, \bar{t})$ and there is a surface roughness $r=\varepsilon^{2} \bar{r}(\bar{x})$. We shall assume the kinematic underheating $\beta$ is of $O(1)$ and the capillary undercooling $\Gamma=\varepsilon^{2} \tilde{\Gamma}$. Because of the difference in accretion rates the water-ice interfacial height in $\bar{x}<\bar{d}_{0}(\bar{t})$ is

$$
\eta=\left\{\begin{array}{cc}
\varepsilon^{2}\left[\bar{r}(\bar{x})+\varepsilon \delta \bar{\eta}_{s}(\bar{x}, \bar{t})\right] & \text { for } S t<1 \\
\varepsilon^{2} \bar{\eta}(\bar{x}, \bar{t}) & \text { for } S t>1
\end{array},\right.
$$

with the difference in scales in (4.1) being due to the enhanced temperature difference in effect for $S t$ greater than unity. It is found that the majority of the flow where $y \sim \varepsilon$ is governed by inviscid dynamics but in addition to inviscid sublayers, where $y \sim \varepsilon^{2}$, viscous and/or thermal wall layers are needed due to the no-slip and wall-temperature conditions. In $\bar{x}<\bar{d}_{0}(\bar{t})$ these are laminar and, as suggested by (3.4)-(3.5), have $y \sim \varepsilon^{3} \delta$ for $S t<1$ and $y \sim \varepsilon^{4} \delta^{2}$ for $S t>1$. The diffusive effects of heat conduction are most important here. 


\subsection{THE INVISCID OUTER REGION}

In the inviscid outer region $I a$ the vertical scale has $y=\varepsilon \bar{y}$ with $\bar{y}=O(1)$ and the velocities and pressure are $u=\bar{u}(\bar{x}, \bar{y}, \bar{t}), v=\bar{v}(\bar{x}, \bar{y}, \bar{t}), p=$ $\varepsilon^{-1} \bar{p}(\bar{x}, \bar{y}, \bar{t})$ to leading-order. The governing equations now reduce to those of unsteady potential flow,

$$
\frac{\partial \bar{u}}{\partial \bar{x}}+\frac{\partial \bar{v}}{\partial \bar{y}}, \quad \frac{\partial \bar{u}}{\partial \bar{t}}=-\frac{\partial \bar{p}}{\partial \bar{x}}, \quad \frac{\partial \bar{v}}{\partial \bar{t}}=-\frac{\partial \bar{p}}{\partial \bar{y}} .
$$

These are subject to the mixed boundary conditions

$$
\begin{aligned}
& \bar{u}=\frac{\partial \bar{Q}}{\partial \bar{x}}(\bar{x}, \bar{t}), \quad \bar{v}=(1-\rho) \frac{\partial \bar{\eta}}{\partial \bar{t}} \quad \text { at } \quad \bar{y}=0^{+} \text {for } \quad|\bar{x}|<\bar{d}_{0}(\bar{t}), \\
& (4.3 a, b) \\
& \bar{p}=0, \quad \bar{v}=\frac{\partial \bar{h}^{+}}{\partial \bar{t}}(\bar{x}, \bar{t}) \quad \text { at } \quad \bar{y}=0^{+} \quad \text { for } \quad|\bar{x}|>\bar{d}_{0}(\bar{t}) .
\end{aligned}
$$

The unknown induced slip velocity and surface pressure are defined by $\bar{u}\left(x, 0^{+}, \bar{t}\right)=\partial \bar{Q} / \partial \bar{x}$ and $\bar{p}\left(x, 0^{+}, \bar{t}\right)=-\partial \bar{Q} / \partial \bar{t}$ respectively. Condition (4.3b) is the kinematic condition on the wetted ice surface, modified to account for a change in density upon solidification, whilst (4.3c) is the Bernoulli condition on the free surface, linearised to apply on the dry region. In addition from (2.4d-f) the initial conditions

$$
h^{+}(\bar{x}, 0)=\bar{h}_{0}(\bar{x}), \quad \bar{\eta}(\bar{x}, 0)=\bar{r}(\bar{x}) \leq \bar{h}_{0}(\bar{x}), \quad \bar{d}_{0}(0)=0,
$$

hold where $\bar{h}_{0}(\bar{x})=\frac{1}{2} \bar{x}^{2}$ and $\bar{r}(0)=0$ along with the far-field conditions

$$
h^{+} \sim \bar{h}_{0}(\bar{x})-\bar{t}, \quad \bar{\eta} \rightarrow 0 \quad \text { as } \quad|x| \rightarrow \infty .
$$

This is consistent with the roughness height tending to zero at large distances. The interaction must also satisfy the Wagner condition, namely

$$
\bar{h}^{+}(\bar{x}, \bar{t})=\bar{\eta}(\bar{x}, \bar{t})=\bar{r}(\bar{x}) \quad \text { at } \quad \bar{x}= \pm \bar{d}_{0}(\bar{t})^{ \pm},
$$

for heights to coincide at the unknown moving contact point which is to be determined. Symmetry of the solution about $\bar{x}=0$ is assumed, while as noted earlier the ice accretion is taken in $(4.3 \mathrm{~g})$ to be comparatively little downstream of the contact point.

The main novelty of the current work is the introduction of the ice accretion rate into the kinematic condition (4.3b) at least for Stefan number $S t>1$ in addition to the presence of roughness; the regime $S t>1$ is of intrinsic interest as well as being relevant to many applications. What we wish to predict are the contact point $x=\bar{d}_{0}(\bar{t})$, the ice shape $\eta(\bar{x}, \bar{t})$ and the 
free surface $\bar{h}^{+}(\bar{x}, \bar{t})$ for a given roughness $\bar{r}(\bar{x})$. As in [57] the solution to (4.2-3) is found to have the form

$$
\frac{\bar{h}^{+}(\bar{x}, \bar{t})-\bar{h}_{0}(\bar{x})}{\left(\bar{x}^{2}-\bar{d}_{0}^{2}(\bar{t})\right)^{\frac{1}{2}}}=-\frac{1}{\pi} \int_{-\bar{d}_{0}(\bar{t})}^{\bar{d}_{0}(\bar{t})} \frac{\bar{h}_{0}(\xi)-(1-\rho) \bar{\eta}(\xi, \bar{t})-\rho \bar{r}(\xi)}{(\bar{x}-\xi)\left(\bar{d}_{0}^{2}(\bar{t})-\xi^{2}\right)^{\frac{1}{2}}} \mathrm{~d} \xi
$$

for $\bar{x}>\bar{d}_{0}(\bar{t})$, which relates the unknown free-surface shape to the unknown accretion shape $\bar{\eta}$ (to be determined below) and contact point $\bar{d}_{0}(\bar{t})$ provided that the physically acceptable condition

$$
h^{+}(\bar{x}, \bar{t}) \geq \bar{r}(x) \text { for } \quad|\bar{x}|>\bar{d}_{0}(\bar{t})
$$

is satisfied. The contact point itself can be determined implicitly from (4.4) by taking the farfield limit of large $\bar{x}$ and using both the asymptotic condition (4.3f) and the symmetry of the configuration to yield

$$
\bar{t}=\frac{2}{\pi} \int_{0}^{\bar{d}_{0}(\bar{t})} \frac{\bar{h}_{0}(\xi)-(1-\rho) \bar{\eta}(\xi, \bar{t})-\rho \bar{r}(\xi)}{\left(\bar{d}_{0}^{2}(\bar{t})-\xi^{2}\right)^{\frac{1}{2}}} \mathrm{~d} \xi .
$$

In particular for the case $S t<1$ or when there is no accretion, and $\bar{\eta}(\bar{x}, \bar{t}) \equiv$ $\bar{r}(\bar{x}),(4.4),(4.6)$ reduce to the forms investigated by [57] for a given roughness $\bar{r}$. Marching forward in time from the square-root solution $\bar{d}_{0}=2 \sqrt{\bar{t}}$ for a smooth wall [57]. obtained solutions for increasingly large values of roughness $\bar{r}$. For sufficiently large roughness forms the condition (4.5) was violated, at which point a new multi-patched solution structure becomes needed. We shall, at least initially, only consider those surface roughnesses which [57] showed gave physically realistic solutions.

The solution (4.4) in general couples the unknown free-surface shape with the unknown accretion shape $\bar{\eta}(\bar{x}, \bar{t})$. Subsequent analysis of the wetted sublayer $I s^{-}$(see $(4.19 c)$ below) yields for $\bar{x}<\bar{d}_{0}(\bar{t})$

$$
\bar{\eta}(\bar{x}, \bar{t})=\bar{r}(\bar{x})+\gamma[\bar{t}-\tau(\bar{x})] \quad \text { for } \quad S t>1
$$

where the constant $\gamma$ is given by (3.3). The time $\tau\left[\bar{d}_{0}(\bar{t})\right]=\bar{t}$ is the inverse function to $\bar{d}_{0}$. Substitution of (4.7a) into (4.4)-(4.6) then yields

$$
\frac{\bar{h}^{+}(\bar{x}, \bar{t})-\bar{h}_{0}(\bar{x})}{\left(\bar{x}^{2}-\bar{d}_{0}^{2}(\bar{t})\right)^{\frac{1}{2}}}=-\frac{1}{\pi} \int_{-\bar{d}_{0}(\bar{t})}^{\bar{d}_{0}(\bar{t})} \frac{\bar{h}_{0}(\xi)-\bar{r}(\xi)-(1-\rho) \gamma[\bar{t}-\tau(\xi)]}{(\bar{x}-\xi)\left(\bar{d}_{0}^{2}(\bar{t})-\xi^{2}\right)^{\frac{1}{2}}} \mathrm{~d} \xi
$$

for $\bar{x}>d_{0}(\bar{t})$, with

$$
\bar{t}=\frac{2}{\pi} \int_{0}^{\bar{d}_{0}(\bar{t})} \frac{\bar{h}_{0}(\xi)-\bar{r}(\xi)-(1-\rho) \gamma[t-\tau(\xi)]}{\left(\bar{d}_{0}^{2}(\bar{t})-\xi^{2}\right)^{\frac{1}{2}}} \mathrm{~d} \xi .
$$


Only for the case $\rho \neq 1$ does ice accretion affect matters, caused by the nonzero normal velocity at the wall. In the absence of roughness, $r \equiv 0$, the simple square-root behaviour for the contact point is retained with

$$
\bar{d}_{0}(\bar{t})=\sqrt{\lambda \bar{t}}=2\left[\left\{1+\frac{1}{2}(1-\rho) \gamma\right\} \bar{t}\right]^{\frac{1}{2}},
$$

so that $\tau(\bar{x})=\bar{x}^{2} / \lambda$, which results in a free-surface shape

$\bar{h}_{0}^{+}(\bar{x}, \bar{t})=\frac{1}{2} \bar{x}\left(\bar{x}^{2}-\bar{d}_{0}^{2}(\bar{t})\right)^{\frac{1}{2}}+(1-\rho) \frac{\gamma}{\lambda}\left[\bar{d}_{0}^{2}(\bar{t})-\bar{x}\left\{\bar{x}-\left(\bar{x}^{2}-\bar{d}_{0}^{2}(\bar{t})\right)^{\frac{1}{2}}\right\}\right]$.

The results $(4.8 \mathrm{~b} a, b)$ are obviously key predictions for a smooth surface. Finally, on approach to the contact point from below, as $\bar{x} \rightarrow \bar{d}_{0}(\bar{t})^{-}$, it can be shown that the effective potential

$$
\bar{Q}(\bar{x}, \bar{t}) \sim-S_{1}(\bar{t})\left[\bar{d}_{0}(\bar{t})-\bar{x}\right]^{\frac{1}{2}}+O\left[\left(\bar{d}_{0}-\bar{x}\right)\right],
$$

where $S_{1}(\bar{t})=\left(2 \bar{d}_{0}\right)^{\frac{1}{2}}$, a result that depends on surface roughness and ice accretion only through the factor $\bar{d}_{0}(\bar{t})$.

\subsection{THE WETTED WALL LAYER FOR SLOW SOLIDIFICATION}

A prime goal here is to determine the thermal behaviour of course but investigation of both flow and temperature is desirable because although the important thermal response is found to uncouple from the flow response the latter forms a significant part of the physics of the multi-structure. For relatively slow accretion let us take $y=\varepsilon^{2}\left[\bar{r}(\bar{x})+\varepsilon \delta \bar{\eta}_{s}(\bar{x}, \bar{t})+\varepsilon \delta \bar{Y}\right]$ in the upstream wetted inner layer $I v^{-}$; then for $\bar{Y}=0(1)$ in $\bar{x}<\bar{d}_{0}$ we expect a solution

$$
\begin{aligned}
& u=\bar{U}(\bar{x}, \bar{Y}, \bar{t}), \quad p=\varepsilon^{-1} \bar{P}(\bar{x}, \bar{Y}, \bar{t}), \quad T=\bar{T}(\bar{x}, \bar{Y}, \bar{t}), \quad(4.10 a-c) \\
& v=\varepsilon \delta(1-\rho) \frac{\partial \bar{\eta}_{s}}{\partial \bar{t}}+\varepsilon\left(\frac{\partial \bar{r}}{\partial \bar{x}}+\varepsilon \delta \frac{\partial \bar{\eta}_{s}}{\partial \bar{x}}\right) \bar{U}+\varepsilon^{2} \delta \bar{V}(\bar{x}, \bar{Y}, \bar{t}), \quad(4.10 d)
\end{aligned}
$$

in view of the orders of magnitude present. At leading order therefore the linearised unsteady viscous/thermal equations apply,

$$
\begin{array}{cc}
\frac{\partial \bar{U}}{\partial \bar{x}}+\frac{\partial \bar{V}}{\partial \bar{Y}}=0, & \frac{\partial \bar{U}}{\partial \bar{t}}-\rho \frac{\partial \bar{\eta}_{s}}{\partial \bar{t}} \frac{\partial \bar{U}}{\partial \bar{Y}}=\frac{\partial \bar{P}}{\partial \bar{x}}+\frac{\partial^{2} \bar{U}}{\partial \bar{Y}^{2}}, \\
0=-\frac{\partial \bar{P}}{\partial \bar{Y}}, & \frac{\partial \bar{T}}{\partial \bar{t}}-\rho \frac{\partial \bar{\eta}_{s}}{\partial \bar{t}} \frac{\partial \bar{T}}{\partial \bar{Y}}=\frac{1}{\operatorname{Pr}} \frac{\partial^{2} \bar{T}}{\partial \bar{Y}^{2}} .
\end{array}
$$

These equations are subject to the classical conditions

$$
\bar{U}=\bar{V}=0, \quad \bar{T}=1, \quad \frac{\rho}{S t} \frac{\partial \bar{\eta}_{s}}{\partial t}=-\frac{1}{\operatorname{Pr}} \frac{\partial \bar{T}}{\partial \bar{Y}} \quad \text { at } \quad \bar{Y}=0, \quad(4.12 a-d)
$$




$$
\bar{U} \rightarrow \frac{\partial \bar{Q}}{\partial \bar{x}}, \quad \bar{T} \rightarrow 0 \quad \text { as } \quad \bar{Y} \rightarrow \infty
$$

Here $(4.12 \mathrm{e}, \mathrm{f})$ ensure a match with the solution in $I a$ provided the unknown pressure $\bar{P}=-\partial \bar{Q} / \partial \bar{t}$. The approach to the asymptotic form (4.12e) for $\bar{U}$ is exponential, ruling out a possible algebraically decaying asymptotic form because the corresponding logarithmic growth in $\bar{V}$ would give rise to anomalous terms of $O\left(\varepsilon^{2} \ln \varepsilon\right)$ in the outer solution. The balance in (4.11d) makes apparent the uncoupling of the thermal response.

Although linear, the presence of the pressure gradient complicates matters with regard to the velocity profile. However, the temperature supports a similarity solution of the form

$$
\bar{T}=g(\hat{\xi})=\frac{\operatorname{erfc}\left[\frac{1}{2} \sqrt{\operatorname{Pr}}(\hat{\xi}+\rho \Lambda)\right]}{\operatorname{erfc}\left[\frac{1}{2} \sqrt{\operatorname{Pr}} \rho \Lambda\right]} \quad \text { where } \quad \hat{\xi}=\frac{\bar{Y}}{\sqrt{\bar{t}-\tau(\bar{x})}}
$$

which satisfies all conditions provided that the solution for $\bar{\eta}_{s}(\bar{x}, \bar{t})$ in $\bar{x}<$ $\bar{d}_{0}(\bar{t})$ is given by

$$
\bar{\eta}_{s}(\bar{x}, \bar{t})=\Lambda \sqrt{\bar{t}-\tau(\bar{x})} \quad \text { where } \quad \tau\left[\bar{d}_{0}(\bar{t})\right]=\bar{t},
$$

and the eigenvalue $\Lambda$ satisfies the classical Stefan eigenrelation (3.2). The solution (4.13b) satisfies the Wagner condition $(4.3 \mathrm{~g}), \bar{\eta}_{s}=0$ at $\bar{x}=\bar{d}_{0}(\bar{t})$ and yields the same square root growth in time $\bar{t}$ at every point $\bar{x}$. The spatial variation arises simply from the time $\tau(\bar{x})$ at which solidification first starts to occur at that point $\bar{x}$, namely when it is the contact point $\bar{x}=\bar{d}_{0}(\tau)$. The absence of viscous influence on the ice growth here is confirmed by the fact that in dimensional terms the ice-water interfacial shape in $(4.13 \mathrm{~b})$ is independent of viscosity.

The solution (4.13b) together with the form of the initiation time $\tau(\bar{x})$ on approaching the turnover point from below suggests that

$$
\bar{\eta}_{s}(\bar{x}, \bar{t})=\Lambda\left(\frac{\mathrm{d} \bar{d}_{0}}{\mathrm{~d} \bar{t}}\right)^{-\frac{1}{2}}\left(\bar{d}_{0}-\bar{x}\right)^{\frac{1}{2}}+O\left[\left(\bar{d}_{0}-\bar{x}\right)^{\frac{3}{2}}\right],
$$

giving a square-root spatial decay in the ice thickness. Compare with (4.20a) below. In addition, the singular behaviour for the pressure $\bar{P} \sim \frac{1}{2} S_{1}(\bar{t}) \mathrm{d} \bar{d}_{0} / \mathrm{d} \bar{t}\left(\bar{d}_{0}-\bar{x}\right)^{-\frac{1}{2}}$ implied by (4.9) suggests a solution

$$
\bar{U}=\frac{1}{2} \frac{S_{1}(\bar{t})}{\left(\bar{d}_{0}-\bar{x}\right)^{\frac{1}{2}}}\left\{\frac{\partial f}{\partial \xi}+O\left(\bar{d}_{0}-\bar{x}\right)^{\frac{1}{2}}\right\}, \quad \bar{T}=g(\xi),
$$

where $\xi=\left(\mathrm{d} \bar{d}_{0} / \mathrm{d} \bar{t}\right)^{\frac{1}{2}}\left(\bar{d}_{0}-\bar{x}\right)^{-\frac{1}{2}} Y, g$ is again given by (4.13a) and the linear similarity equation for $f$ has

$$
f(\xi)=\xi-\sqrt{\pi} \mathrm{e}^{\frac{1}{4}(\rho \Lambda)^{2}}\left\{\operatorname{erfc}\left[\frac{1}{2}(\xi+\rho \Lambda)\right]-\operatorname{erfc}\left(\frac{1}{2} \rho \Lambda\right)\right\} .
$$


The response (4.14) can be shown to yield the scales for both the fairly passive intermediate inner region $I I v^{-}$, when $\bar{d}_{0}-\bar{x}$ is $O(\varepsilon)$, and the viscous region IIIv when $\bar{d}_{0}-\bar{x}$ is $O\left(\varepsilon^{2}\right)$. These regions are treated as distinct because of their very different vertical and horizontal extents shown in figures 2,3 effectively.

\subsection{THE WETTED WALL LAYER DURING FAST SOLIDIFICATION}

Here as in the previous sub-section a prime goal is to determine the temperature response. In relatively fast accretion the wetted wall layer is $\mathrm{Is}^{-}$ upstream. We take $y=\varepsilon^{2}\left[\bar{\eta}(\bar{x}, \bar{t})+\varepsilon^{2} \delta^{2} \tilde{Y}\right]$ and then for $\tilde{Y}=0(1)$ in $\bar{x}<\bar{d}_{0}$ we propose a solution

$$
\begin{gathered}
u=\bar{U}(\bar{x}, \tilde{Y}, \bar{t}), \quad p=\varepsilon^{-1} \bar{P}(\bar{x}, \tilde{Y}, \bar{t}), \quad T=\bar{T}(\bar{x}, \tilde{Y}, \bar{t}), \quad(4.16 a-c) \\
v=(1-\rho) \frac{\partial \bar{\eta}}{\partial \bar{t}}+\varepsilon \frac{\partial \bar{\eta}}{\partial \bar{x}} \bar{U}+\varepsilon^{3} \delta^{2} \bar{V}(\bar{x}, \tilde{Y}, \bar{t}) .
\end{gathered}
$$

At leading order now the linearised boundary-layer and thermal equations apply,

$$
\frac{\partial \bar{U}}{\partial \bar{x}}+\frac{\partial \bar{V}}{\partial \tilde{Y}}=0, \quad-\rho \frac{\partial \bar{\eta}}{\partial \bar{t}} \frac{\partial \bar{U}}{\partial \bar{Y}}=\frac{\partial^{2} \bar{U}}{\partial \tilde{Y}^{2}}, \quad-\rho \frac{\partial \bar{\eta}}{\partial \bar{t}} \frac{\partial \bar{T}}{\partial \tilde{Y}}=\frac{1}{\operatorname{Pr}} \frac{\partial^{2} \bar{T}}{\partial \tilde{Y}^{2}},
$$

with the temperature uncoupled from the flow equations again. The system is subject to the non-classical (undercooling) conditions

$$
\begin{gathered}
\bar{U}=\bar{V}=0, \quad \bar{T}=1-\beta \frac{\partial \eta}{\partial t}, \quad \text { at } \quad \tilde{Y}=0, \quad(4.18 a-c) \\
{\left[\frac{\rho}{S t}-\left(1-\rho c_{p}\right) \beta \frac{\partial \eta}{\partial t}\right] \frac{\partial \eta}{\partial t}=-\frac{1}{\operatorname{Pr}} \frac{\partial \bar{T}}{\partial \tilde{Y}} \quad \text { at } \quad \tilde{Y}=0} \\
\bar{U} \rightarrow \frac{\partial \bar{Q}}{\partial \bar{x}}, \quad \bar{T} \rightarrow 0 \quad \text { as } \quad \tilde{Y} \rightarrow \infty
\end{gathered}
$$

where $(4.18 \mathrm{e}, \mathrm{f})$ ensure a match with the outer flow in $I a, b$. We obtain the solution

$$
\bar{U}=\frac{\partial \bar{Q}}{\partial \bar{x}}\left[1-\exp \left(-\rho \frac{\partial \bar{\eta}}{\partial \bar{t}} \tilde{Y}\right)\right], \quad \bar{T}=\left(1-\beta \frac{\partial \eta}{\partial t}\right) \exp \left(-\operatorname{Pr} \rho \frac{\partial \bar{\eta}}{\partial \bar{t}} \tilde{Y}\right),
$$

which holds provided that the solution for $\bar{\eta}(\bar{x}, \bar{t})$ in $\bar{x}<\bar{d}_{0}(\bar{t})$ is given by

$$
\eta(\bar{x}, \bar{t})=\bar{r}(\bar{x})+\gamma[\bar{t}-\tau(\bar{x})],
$$

and the constant $\gamma$ is given by (3.3). The independence of the ice growth from any viscous influence here during fast solidification is similar to that for 
slow solidification. Here (4.19c) satisfies the Wagner condition (4.3g), which here is $\bar{\eta}_{0}=\bar{r}\left[d_{0}(\bar{t})\right]$ at $\bar{x}=\bar{d}_{0}(\bar{t})$, and the ice accretes linearly in time $\bar{t}$. This contrasts with the result $(4.13 b)$ of slow accretion. The most relevant comments regarding the spatial variation are nevertherless exactly the same as in the previous section.

The shape behaviour (4.19c) together with the form of $\tau(\bar{x})$ now implies that

$$
\bar{\eta}(\bar{x}, \bar{t})-\bar{r}(\bar{x}) \sim \gamma\left(\frac{\mathrm{d} \tilde{d}_{0}}{\mathrm{~d} \tilde{t}}\right)^{-1}\left(\bar{d}_{0}-\bar{x}\right)+O\left[\left(\bar{d}_{0}-\bar{x}\right)^{2}\right],
$$

so that we have a linear decay in the ice thickness. From (4.9) and the solution $(4.19 \mathrm{a}-\mathrm{c})$ this leads to the local velocity and thermal distributions

$$
\begin{gathered}
\bar{U}=\frac{1}{2} \frac{S_{1}(\bar{t})}{\left(\bar{d}_{0}-\bar{x}\right)^{\frac{1}{2}}}\left\{1+O\left(\bar{d}_{0}-\bar{x}\right)^{\frac{1}{2}}\right\}[1-\exp (-\rho \gamma \tilde{Y})], \\
\bar{T}=(1-\beta \gamma) \exp (-\operatorname{Pr} \rho \gamma \tilde{Y}) .
\end{gathered}
$$

The behaviour in $(4.20 \mathrm{a}, \mathrm{b})$ yields the scales for the passive intermediate region $I I s^{-}$when $\bar{d}_{0}-\bar{x}$ is $O(\varepsilon)$, as well as those of the inner region IIIs when $\bar{d}_{0}-\bar{x}$ is $O\left(\varepsilon^{2}\right)$. Again the regions are distinct as given in figures 2,3 in schematic form.

\subsection{THE DRY-SIDE SPLASH JET AND WALL LAYER}

As discussed previously, our analysis does not need to give any indication of the precise size of the ice accretion in $\bar{x}>\bar{d}_{0}(\bar{t})$, i.e. downstream on the dry-side. Nevertheless some further insights into both the dry-side splash jet and wall layer are required for the multi-structure and to help provide a proper context subject to the likely complex flow properties there which are described just after (3.6). We recall that $\eta-r=O\left(\varepsilon^{3} \delta\right)$ for $S t<1$ and $\eta-r=O\left(\varepsilon^{2} / \beta\right)$ for $S t>1$ upstream of the contact point and $\eta-$ $r=O\left(\varepsilon^{4} \delta\right)$ or $O\left(\varepsilon^{4} / \beta\right)$ respectively in the immediate vicinity of the contact point. Let us make the tentative assumption that $\eta=\varepsilon^{2}\left[\bar{r}(\bar{x})+\varepsilon \delta \bar{\eta}^{R}(\bar{x}, \bar{t})\right]$, with roughness given by $r=\varepsilon^{2} \bar{r}(\bar{x})$ and with $h^{-}=\varepsilon^{2}\left[\bar{r}(\bar{x})+\varepsilon \bar{h}^{-}(\bar{x}, \bar{t})\right]$ being the splash jet thickness. Four points then stand out.

First, the dry-side wall layer $I v^{+}$then has $y=\varepsilon^{2}\left[\bar{r}(\bar{x})+\varepsilon \delta \bar{\eta}^{R}(\bar{x}, \bar{t})+\varepsilon \delta \bar{Y}\right]$ for $\bar{Y}=O(1)$ in $\bar{x}>\bar{d}_{0}$ and a solution

$$
\begin{gathered}
u=\varepsilon^{-1} \bar{U}(\bar{x}, \bar{Y}, \bar{t}), \quad p=\varepsilon^{-2} \bar{P}(\bar{x}, \bar{Y}, \bar{t}), \quad T=\bar{T}(\bar{x}, \bar{Y}, \bar{t}), \quad(4.21 a-c) \\
v=\varepsilon \delta(1-\rho) \frac{\partial \bar{\eta}^{R}}{\partial \bar{t}}+\left(\frac{\partial \bar{r}}{\partial \bar{x}}+\varepsilon \delta \frac{\partial \bar{\eta}^{R}}{\partial \bar{x}}\right) \bar{U}+\varepsilon \delta \bar{V}(\bar{x}, \bar{Y}, \bar{t}) .
\end{gathered}
$$


At leading order the nonlinear unsteady boundary-layer equations therefore apply,

$$
\begin{gathered}
\frac{\partial \bar{U}}{\partial \bar{x}}+\frac{\partial \bar{V}}{\partial \bar{Y}}=0 \\
\frac{\partial \bar{U}}{\partial \bar{t}}+\bar{U} \frac{\partial \bar{U}}{\partial \bar{x}}+\left(\bar{V}-\rho \frac{\partial \bar{\eta}^{R}}{\partial \bar{t}}\right) \frac{\partial \bar{U}}{\partial \bar{Y}}=-\frac{\partial \bar{P}}{\partial \bar{x}}+\frac{\partial^{2} \bar{U}}{\partial \bar{Y}^{2}}, \quad 0=-\frac{\partial \bar{P}}{\partial \bar{Y}} \\
\frac{\partial \bar{T}}{\partial \bar{t}}+\bar{U} \frac{\partial \bar{T}}{\partial \bar{x}}+\left(\bar{V}-\rho \frac{\partial \bar{\eta}^{R}}{\partial \bar{t}}\right) \frac{\partial \bar{T}}{\partial \bar{Y}}=\frac{1}{\operatorname{Pr}} \frac{\partial^{2} \bar{T}}{\partial \bar{Y}^{2}}
\end{gathered}
$$

The main boundary conditions are

$$
\begin{aligned}
\bar{U}=\bar{V} & =0, \quad \bar{T}=1, \quad \frac{\rho}{S t} \frac{\partial \bar{\eta}^{R}}{\partial \bar{t}}=-\frac{1}{\operatorname{Pr}} \frac{\partial \bar{T}}{\partial \bar{Y}} \quad \text { at } \quad \bar{Y}=0, \quad(4.23 a-d) \\
\bar{U} & \rightarrow U^{*}(\bar{x}), \quad \bar{T} \rightarrow 0, \quad \bar{P} \rightarrow 0 \quad \text { as } \quad \bar{Y} \rightarrow \infty . \quad(4.23 e-g)
\end{aligned}
$$

Ice-shape displacement is effective in $(4.22 b)$ but there is an absence of any kinetic undercooling contribution in $(4.23 \mathrm{~b}, \mathrm{c})$ due to the relative smallness of the accreted ice shape on the dry side. Also the fact that $\partial \bar{P} / \partial \bar{Y}=0$ implies $\bar{P} \equiv 0$. Indeed (second point) from the inviscid splash-jet solution for the region $I c$ just outside the present layer the slip velocity satisfies the zero-gravity shallow-water equations

$$
\frac{\partial U^{*}}{\partial \bar{t}}+U^{*} \frac{\partial U^{*}}{\partial \bar{x}}=0, \quad \frac{\partial \bar{h}^{-}}{\partial \bar{t}}+\frac{\partial}{\partial \bar{x}}\left(U^{*} \bar{h}^{-}\right)=0
$$

where the first equation also follows from the asymptotic form (4.23e). Matching to the inner inviscid region II I a yields [48]

$$
U^{*}=2 \frac{\mathrm{d} \bar{d}_{0}}{\mathrm{~d} \bar{t}}, \quad \bar{h}^{-}(\bar{x}, \bar{t})=H_{J}(\bar{t}) \equiv \frac{1}{8} \pi \bar{d}_{0}(\bar{t})\left(\frac{\mathrm{d} \bar{d}_{0}}{\mathrm{~d} \bar{t}}\right)^{-1}
$$

at $\bar{x}=\bar{d}_{0}(\bar{t})$. The first-order hyperbolic equation (4.24a) can be solved by characteristics to give

$$
U^{*}=2 \frac{\mathrm{d} \bar{d}_{0}}{\mathrm{~d} s} \quad \text { on } \quad \bar{t}=s+\frac{1}{2}\left(\frac{\mathrm{d} \bar{d}_{0}}{\mathrm{~d} s}\right)^{-1}\left[\bar{x}-\bar{d}_{0}(s)\right]
$$

for some parameter $s>0$, with the solution of (4.24b) for $\bar{h}^{-}$following once the form of $\bar{d}_{0}$ and $U^{*}$ is established. It is interesting that the splashjet solution $(4.25 \mathrm{a}, \mathrm{b})$ depends on ice accretion and surface roughness only through the form of $\bar{d}_{0}(\bar{t})$. In the absence of roughness, where $\bar{d}_{0}=\sqrt{\lambda \bar{t}}$ from (4.8a), we can infer that $H_{J}(\bar{t})=\frac{1}{2}(\pi / \lambda) \bar{t}^{\frac{3}{2}}$ together with

$$
U^{*}(\bar{x}, \bar{t})=\frac{\bar{x}}{\bar{t}}, \quad \bar{h}^{-}(\bar{x}, \bar{t})=\frac{1}{2} \pi \lambda^{2} \bar{t}^{4} \bar{x}^{5},
$$


which yields a splash jet that is infinitely long, constantly accelerating and thinning. In the absence of roughness ice accretion tends to slightly speed up and thin the splash jet. The interaction between these two effects is discussed later.

Third, concerning the splash jet further, clearly shocks (hydraulic jumps) are avoided when $\bar{d}_{0}^{\prime \prime}(\bar{t})<0$, and there is no intersecting of different straightline characteristic traces, or multiple intersection of the data curve $\bar{x}=\bar{d}_{0}(\bar{t})$ by a single trace. Although this clearly is the case in the absence of roughness, the presence of roughness makes a similar statement necessarily more difficult. In their investigation [57] did not consider the splash jet but were concerned when roughness caused $\bar{d}_{0}^{\prime}(\bar{t}) \rightarrow+\infty$, which was then usually followed by an unphysical period during which $\bar{d}_{0}^{\prime}(\bar{t})<0$, both being an indication of a second impact, or touchdown with (4.5) no longer satisfied. However before this occurs we must have $\bar{d}_{0}^{\prime \prime}(\bar{t}) \geq 0$ arising. Thus it is possible that roughness leads to hydraulic jumps in the splash jet prior to touchdown, a matter investigated in detail in the next section.

Fourth, close to the contact point as $\bar{x} \rightarrow \bar{d}_{0}(\bar{t})^{+}$the flow solution to the boundary-layer problem (4.22)-(4.24) with a laminar assumption takes on a two-tiered subform. (The tentative nature of the assumption should be noted, given that complex flow is expected here.) Firstly there is an outer inviscid tier for $\bar{Y}=O(1)$, where $\bar{U}=U^{*}(\bar{x}, \bar{t}) \approx 2 \mathrm{~d} \bar{d}_{0} / \mathrm{d} \bar{t}, \bar{T} \equiv 0$ corresponding to uniform temperature. Secondly, for $\bar{Y}=O\left(\bar{x}-\bar{d}_{0}\right)^{-\frac{1}{2}}$ there is an inner Blasius-like viscous tier with

$$
\begin{gathered}
\bar{U}=\left(\frac{\mathrm{d} \bar{d}_{0}}{\mathrm{~d} \bar{t}}\right)\left\{\left(1+\frac{\partial f_{B}}{\partial \xi}\right)+O\left[\left(\bar{x}-\bar{d}_{0}\right)^{\frac{1}{2}}\right]\right\}, \quad \bar{T}=g_{B}(\xi), \\
\bar{V}=\frac{\left(\mathrm{d} \bar{d}_{0} / \mathrm{d} \bar{t}\right)^{\frac{1}{2}}}{2\left(\bar{x}-\bar{d}_{0}\right)^{\frac{1}{2}}}\left\{\left(\xi \frac{\partial f_{B}}{\partial \xi}-f_{B}-\rho \Lambda^{+}\right)+O\left[\left(\bar{x}-\bar{d}_{0}\right)^{\frac{1}{2}}\right]\right\},
\end{gathered}
$$

where $\xi=\left(\mathrm{d} \bar{d}_{0} / \mathrm{d} \bar{t}\right)^{\frac{1}{2}}\left(\bar{d}_{0}-\bar{x}\right)^{-\frac{1}{2}} Y$, and

$$
\bar{\eta}^{R}(\bar{x}, \bar{t})=L_{0}(\bar{t})+\Lambda^{+}\left(\frac{\mathrm{d} \bar{d}_{0}}{\mathrm{~d} \bar{t}}\right)^{-\frac{1}{2}}\left(\bar{x}-\bar{d}_{0}\right)^{\frac{1}{2}}+O\left[\left(\bar{x}-\bar{d}_{0}\right)\right],
$$

for some unknown constant $\Lambda^{+}$. Here $L_{0}(\bar{t})$ appears arbitrary, the temperature profile is unknown and $f_{B}, g_{B}$ satisfy the similarity equations

$$
f_{B}^{\prime \prime \prime}+\frac{1}{2} f_{B} f_{B}^{\prime \prime}=0, \quad \frac{1}{\operatorname{Pr}} g_{B}^{\prime \prime}+\frac{1}{2} f_{B} g_{B}^{\prime}=0,
$$

subject to

$$
\begin{gathered}
f_{B}(0)=-\rho \Lambda^{+}, \quad f_{B}^{\prime}(0)=-1 \\
g_{B}(0)=1, \quad g_{B}^{\prime}(0)=\frac{1}{2} \Lambda^{+} \frac{\operatorname{Pr} \rho}{S t}
\end{gathered}
$$




$$
f_{B}^{\prime}(\infty)=1, \quad g_{B}(\infty)=0 .
$$

Thus $f_{B}(\xi)$ satisfies the Blasius similarity equation (4.27a) or (3.6) subject to constraints equivalent to a boundary layer having a uniform outer flow past a reversed moving wall of equal uniform speed which is also subject to wall blowing for $\Lambda^{+} \geq 0$. (There appears to be no solution of the above assumed laminar form, thus confirming a more complex form is present as mentioned in $\$ 3.3$ earlier.) The assumed behaviour (4.26) near $\bar{x}=\bar{d}_{0}$ is in keeping with the rest of the multi-structure, matching to both the intermediate region $I I v^{+}$ when $\bar{x}-\varepsilon \bar{d}_{0}$ is $O\left(\varepsilon^{2}\right)$ and the inner region IIIv when $\bar{x}-\varepsilon \bar{d}_{0}$ is $O\left(\varepsilon^{3}\right)$.

\section{Numerical findings}

The results in this section are for Stefan numbers St greater than unity, which is a range of some current concern and is fruitful in allowing some explicit results. Solutions were computed for a given roughness shape $\bar{r}(\bar{x})$ which was assumed to be symmetric about $\bar{x}=0$, meaning that only the solution in $\bar{x} \geq 0$ need be considered. Here we follow [57] and consider the specific forms

$$
\bar{r}(\bar{x})=r_{1} \mathrm{e}^{-r_{2}\left(\bar{x}-r_{3}\right)^{2}}-\mathrm{e}^{-r_{2} r_{3}^{2}} \text { for } 0 \leq \bar{x} \leq \bar{a}
$$

where $r_{1}, r_{2}$ and $r_{3}$ are constants. The specific solutions are believed to show the fundamental general points. A sufficiently large value of $r_{2} r_{3}^{2}$ is taken to ensure that the induced surface slope is negligible at $\bar{x}=0$ and $\bar{r}$ decays considerably downstream of the maximum point $r_{3}$ before being reduced fairly smoothly towards zero downstream of $\bar{a}$ which has a large positive prescribed value taken equal to 16 in every case. Since $\bar{r}(\bar{x})$ tends to zero for large $\bar{x}$ downstream we must have $\bar{d}_{0}(\bar{t}) \sim \sqrt{\lambda \bar{t}}$ for large times $\bar{t}$ where $\lambda$ is given by $(4.8 a)$. All calculations took $\rho=0.9$ and differing values of $\gamma$, defined by (3.3), so that $K=(1-\rho) \gamma$ took the values $0,0.5,1.0,1.5$ and 2.0. We note that large values of $\gamma$ correspond to small $\beta$ values.

Equation (4.7c) is first solved by quadrature to obtain the relation $\bar{t}=$ $\tau\left(\bar{d}_{0}\right)$, which is then inverted to give the solution $\bar{d}_{0}(\bar{t})$. Substitution into (4.7a) then gives the shape of the ice surface whereas (4.7b) yields the shape of the free surface.

In the first case a mild roughness shape is considered where $r(x)$ is given by (5.1) with $r_{1}=18, r_{2}=0.124$ and $r_{3}=8$. Figure 4 shows the plot of the contact point $\bar{d}_{0}(\bar{t})$ and its speed $\bar{d}_{0}^{\prime}(\bar{t})$ and figure 5 (a)-(d) show profiles of the free-surface shape for various fixed values of $\bar{d}_{0}(\bar{t})$. It was found that the larger the value of $\gamma$, the quicker the contact point reached a given station. It can be seen that both the ice shape and the free-surface shape deform in the vicinity of the contact point. For this case the entire surface can be wetted. In more detail results for various values of the scaled solidification-growth 

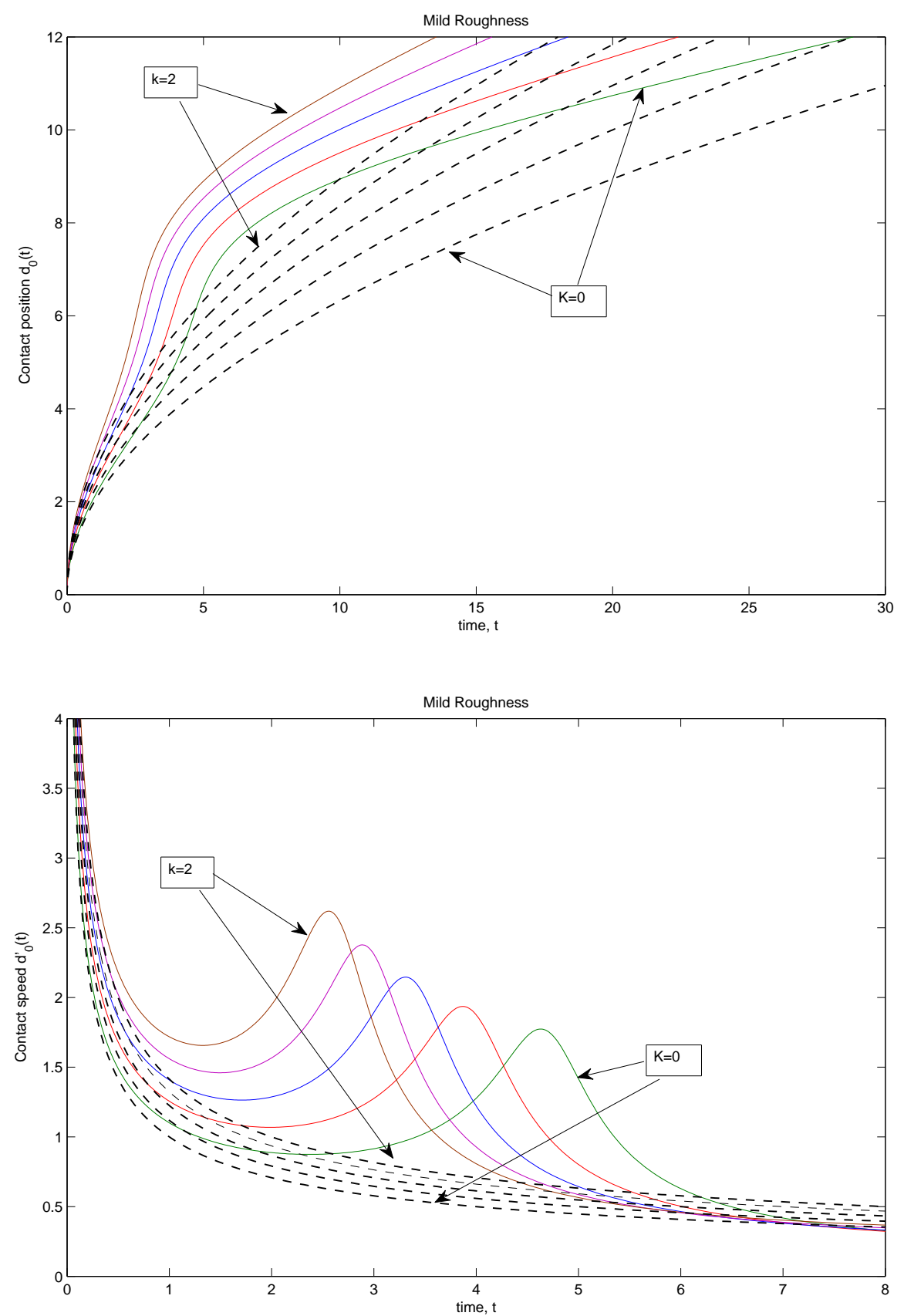

Figure 4. The contact point position $\bar{d}_{0}(\bar{t})$ [on the left] and speed $\bar{d}_{0}^{\prime}(\bar{t})$ [on the right] for a relatively shallow surface roughness, for $K=(1-\rho) \gamma=0,0.5,1.0,1.5,2.0$. The dashed curves denote the analytic form (4.8a) without roughness. 

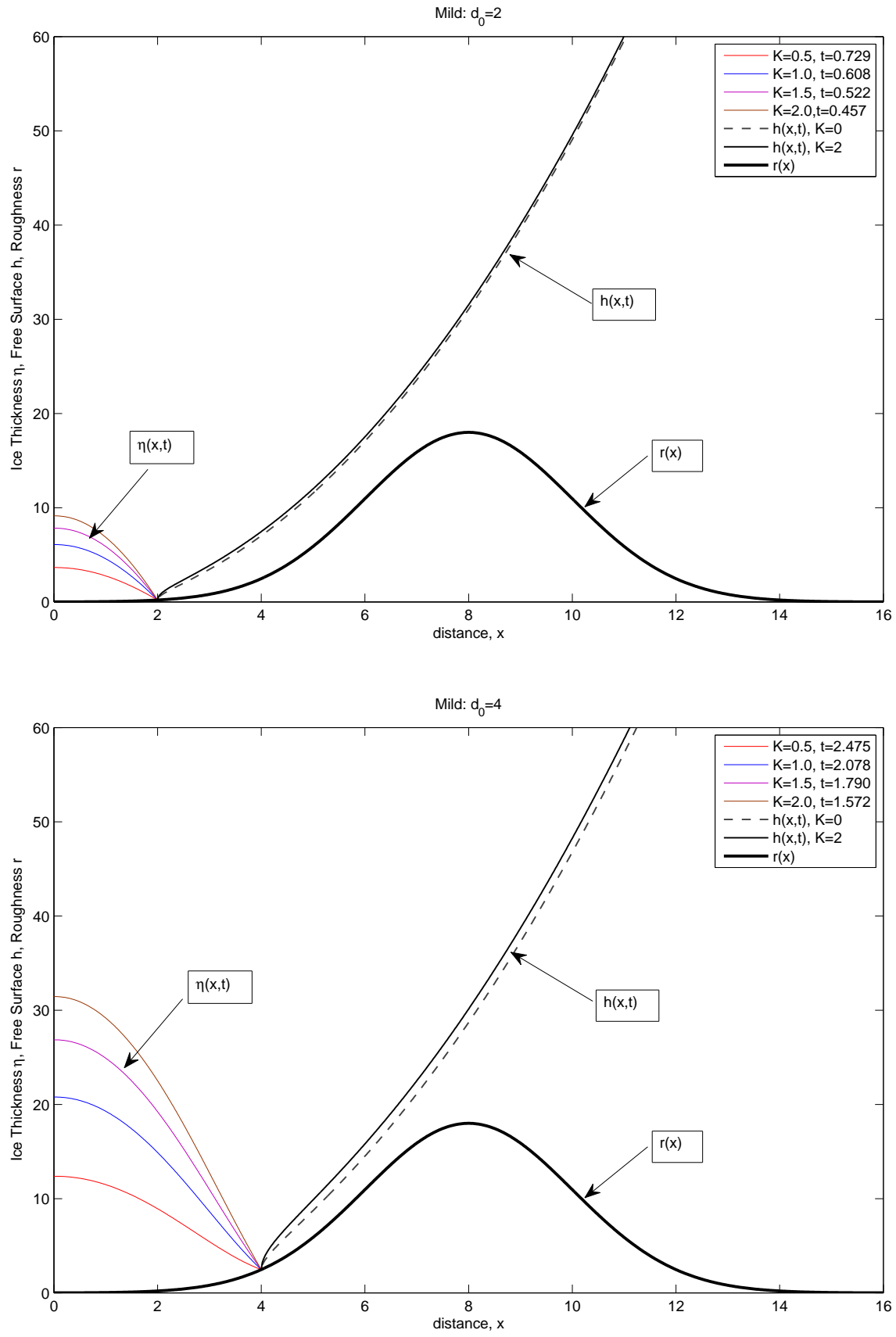

Figure 5. The ice thickness $\bar{\eta}$, free surface $\bar{h}$ and comparatively shallow surface roughness $\bar{r}$ as in figure 4 , when $\bar{d}_{0}=2,4,6$ and 8 , for $K=(1-\rho) \gamma=0,0.5,1,1.5$ and 2 . 

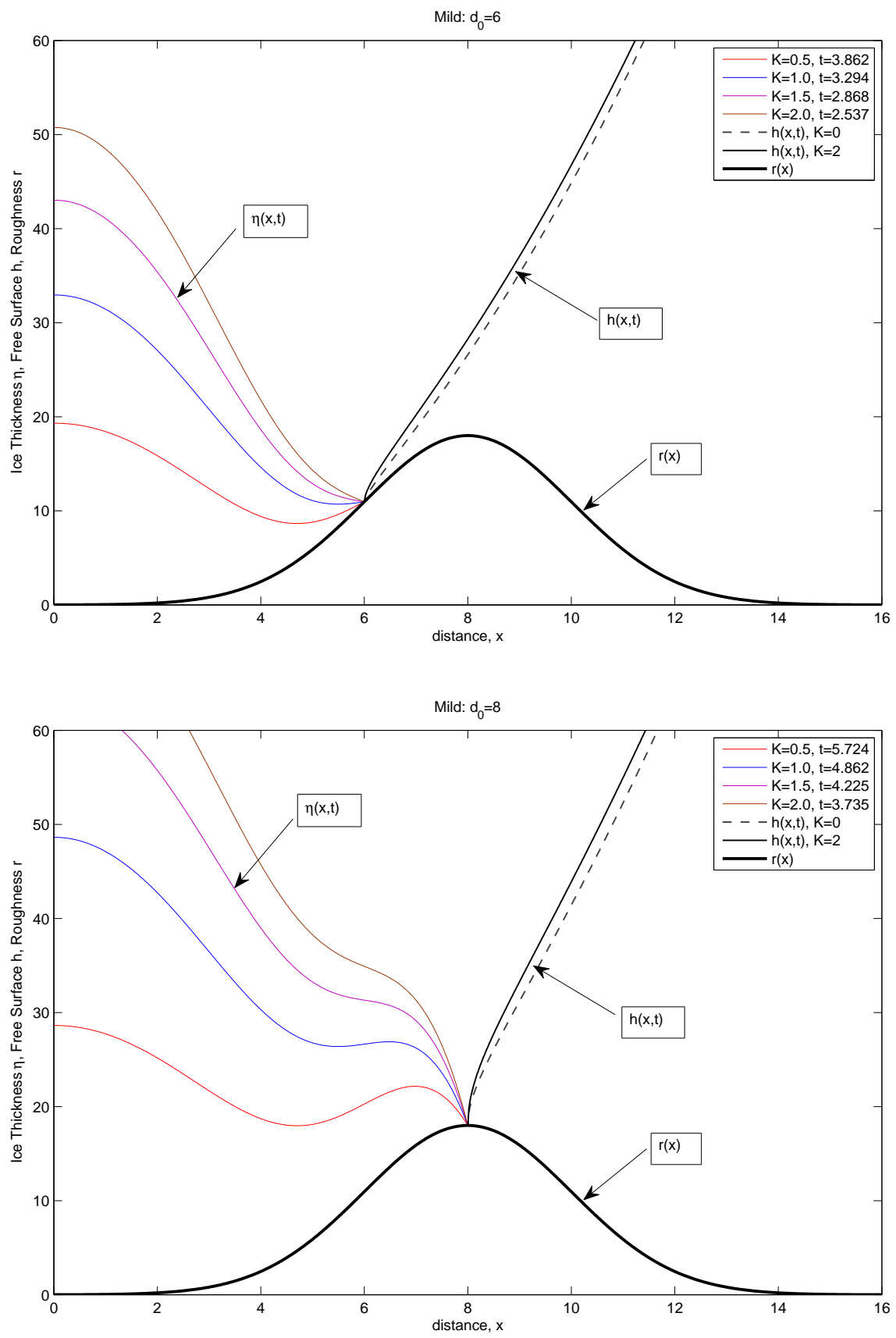

Figure 5. The ice thickness $\bar{\eta}$, free surface $\bar{h}$ and comparatively shallow surface roughness $\bar{r}$ as in figure 4 , when $\bar{d}_{0}=2,4,6$ and 8 , for $K=(1-\rho) \gamma=0,0.5,1,1.5$ and 2 . 
parameter $K=(1-\rho) \gamma$ are explored in figure 4. The progress of the contact point is monotonic in time for the mild roughness and the speed of movement increases with increasing values of the parameter with a noticeable peak arising in the speed as plotted on the right in the figure. The broad trend shows roughness delaying the contact point a little, apart from some exceptions. In figure 5 (a)-(d) there is no ice initially when the contact point is at a scaled distance of zero of course but depending on $\gamma$ the ice first forms on the left behind the contact point and is parabolic in shape by the time the scaled distance reaches 2 . Inflectional ice profiles emerge between the distances of 2 and 4 and become more pronounced at distance 6 , as the distortion of the free-surface shape then becomes clear above the roughness prior to the free surface riding over the top of the roughness.

In the second case a steep roughness shape is taken which again has the Gaussian form (5.1) but here $r_{1}=22, r_{2}=1.125$ and $r_{3}=8$. Figure 6 shows a plot of the contact motion and its speed. As found by Ellis et al, in the absence of icing $(\gamma=0)$ the contact point moves over the steep surface only until a stage where it appears as if the contact point suddenly moves backwards in time, a physically unrealistic situation. The explanation for this is provided by figure 7 (a)-(d) which gives the ice and free-surface shapes. At some point prior to the unphysical behaviour arising a second touchdown occurs near the top of the roughness. The addition of icing causes a reduction in the nonphysical motion of the contact point. The effect of this is that the contact point can move further along the roughness before the solution based on the formal use of (4.7c) breaks down. Indeed it becomes feasible that for a given roughness interactive solutions which terminate without icing can continue to hold with sufficient icing that the entire surface is wetted.

To complete the calculations is the marginal case in which a second touchdown of the droplet nearly occurs in the absence of icing. The marginal case has the values $r_{1}=22, r_{2}=0.1125$ and $r_{3}=8$ and its results are given in figures 8 and 9 . The contact-point motion in figure 8 shows trends resembling those in figure 6. Likewise in figure 9 (a)-(d) the ice growth is broadly similar to that observed in figure 4 apart from the enhanced inflectional shapes found at scaled distances of 4,6 . The behaviour of the free surface is also broadly similar except for the nearly tangential departure seen in figure 8(c).

Ellis et al. derived a condition to determine whether a second touchdown occurs or not. Their first suggestion, namely that a solution is physically valid until $\bar{d}_{0}^{\prime} \rightarrow \infty$, was rejected because it corresponds to an infinite speed of the contact point, which runs counter to the neglect of compressibility effects. Moreover their results established that an impact actually occurs when the fluid surface lies tangentially across the rough surface, forming a contact line rather than a contact point. This ensures that as the wetting continues the 

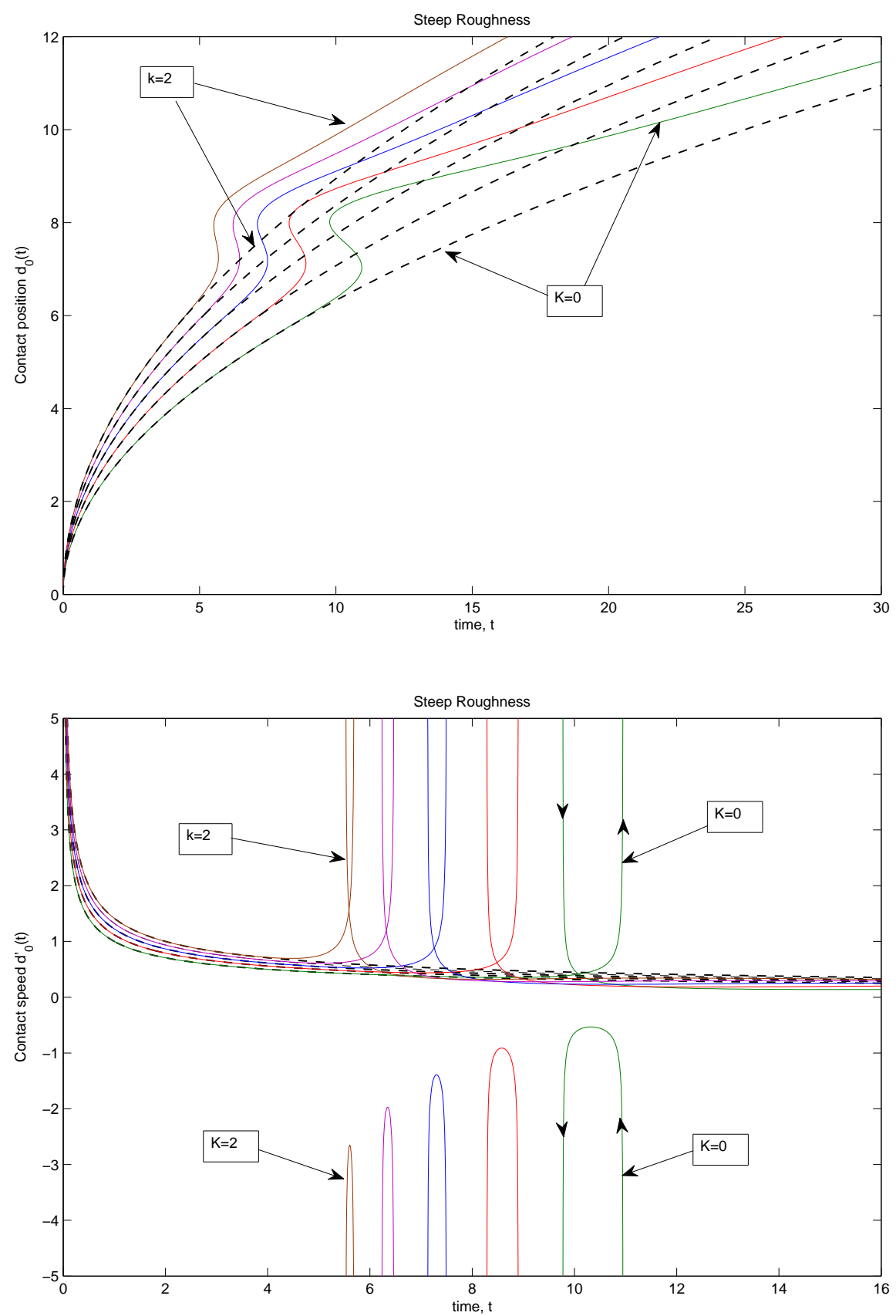

Figure 6. The contact point position $\bar{d}_{0}(\bar{t})$ and speed $\bar{d}_{0}^{\prime}(\bar{t})$ for a comparatively steep surface roughness, with $K=(1-\rho) \gamma=0,0.5,1.0,1.5,2.0$. The dashed curves denote the analytic form (4.8a) without roughness. A breakdown or unphysical response is emphasized by the discontinuous results on the right, the arrows indicating the implied time reversal obtained. 

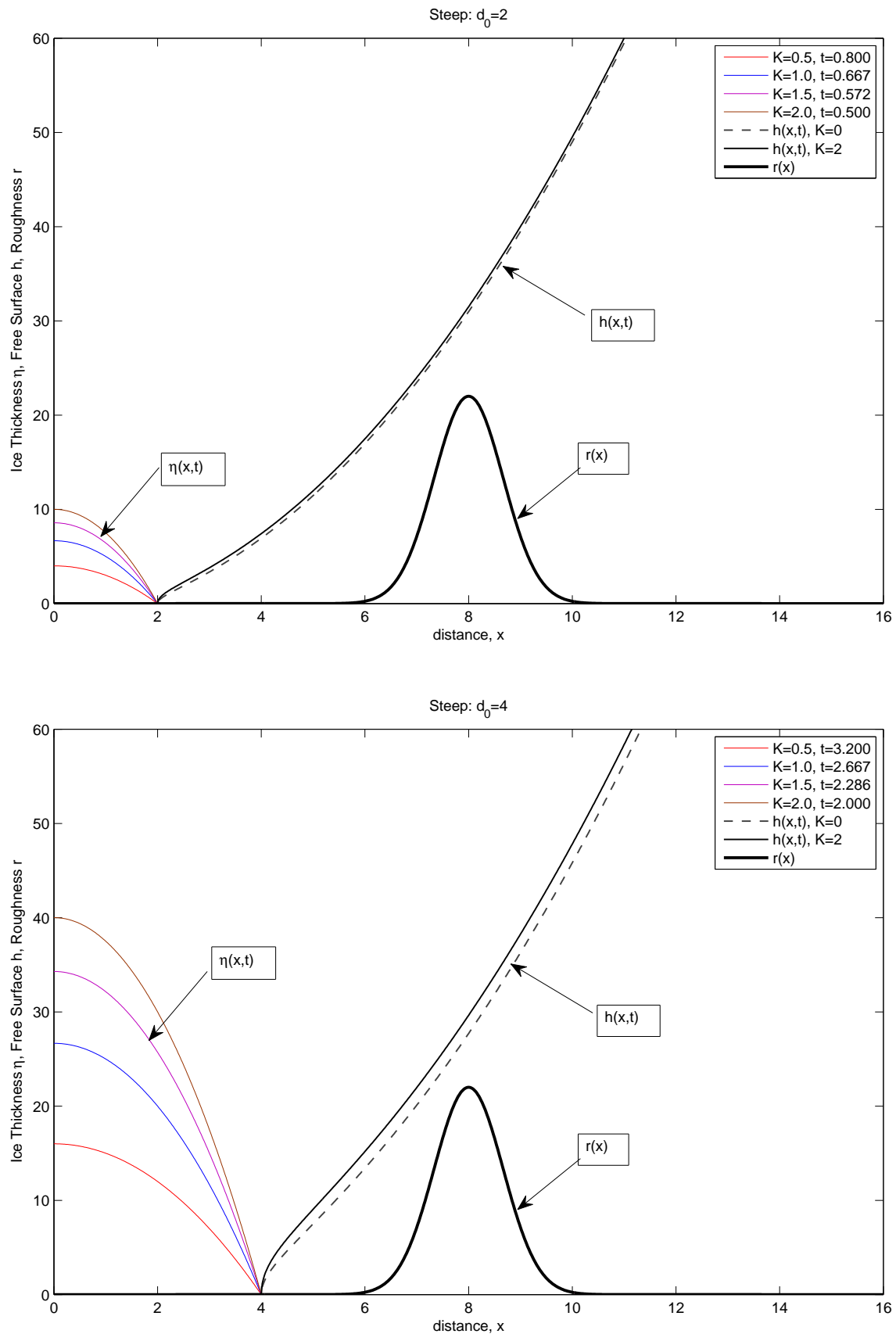

Figure 7. The ice thickness $\bar{\eta}$, free surface shape $\bar{h}$ and relatively steep surface roughness $\bar{r}$ as in figure 6 , when $\bar{d}_{0}=2,4,6$ and 6.5 , for $K=(1-\rho) \gamma=0,0.5,1,1.5$ and 2. A second touchdown occurs only for $K=0$ and 0.5 at $\bar{d}_{0}=6.0$, but for all cases at $\bar{d}_{0}=6.5$. 

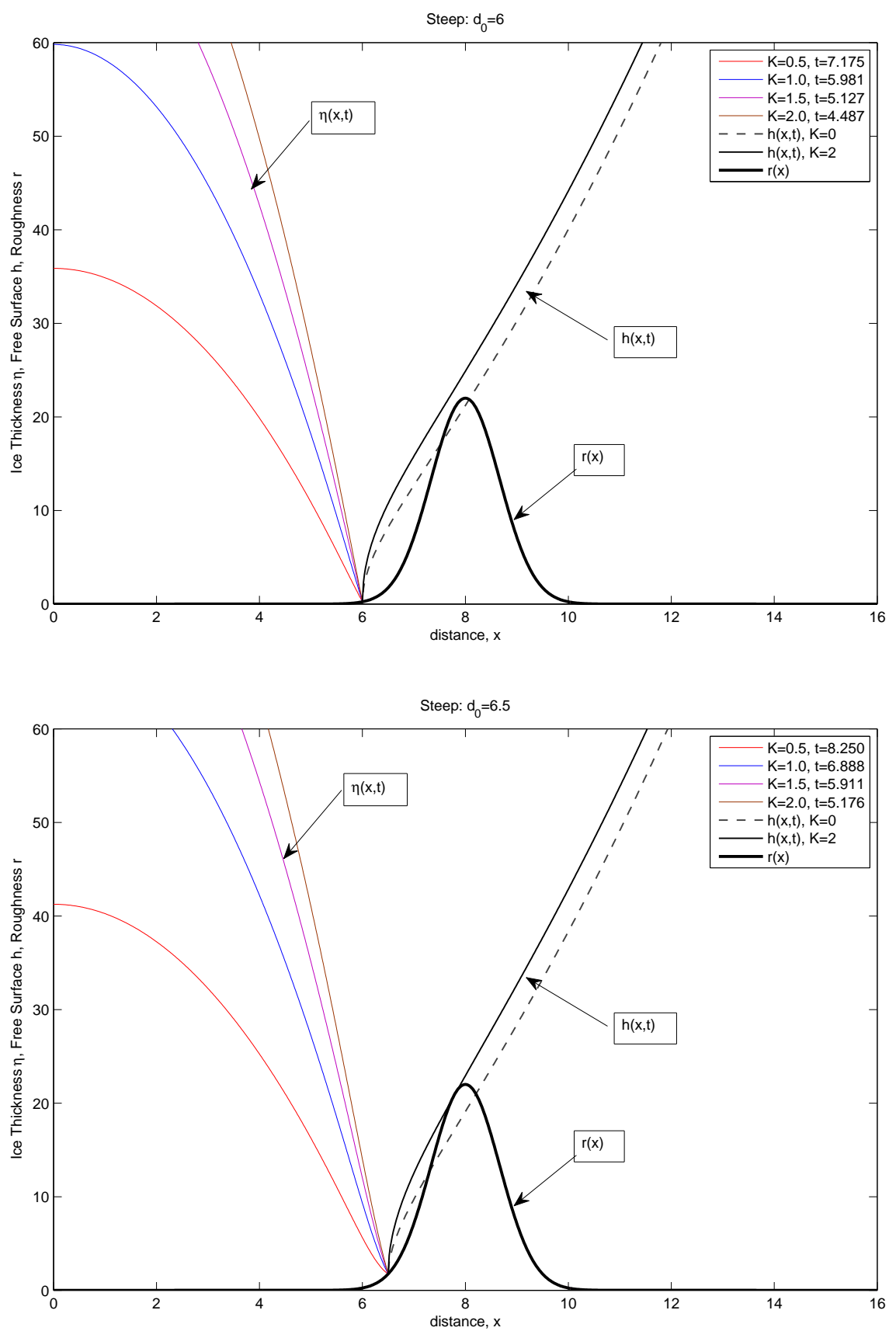

Figure 7. The ice thickness $\bar{\eta}$, free surface shape $\bar{h}$ and relatively steep surface roughness $\bar{r}$ as in figure 6 , when $\bar{d}_{0}=2,4,6$ and 6.5 , for $K=(1-\rho) \gamma=0,0.5,1,1.5$ and 2 . A second touchdown occurs only for $K=0$ and 0.5 at $\bar{d}_{0}=6.0$, but for all cases at $\bar{d}_{0}=6.5$. 
contact point motion resumes from the far end of the contact line, causing a sudden jump in the position of the contact point.

The calculations show that surface roughness alone without additional icing is sufficient to alter the nature of the contact speed $\bar{d}_{0}^{\prime}(\bar{t})$. Even for the mild case (figure 4) both a local minimum and maximum in $\bar{d}_{0}^{\prime}(\bar{t})$ arise, implying a change in sign in $\bar{d}_{0}^{\prime \prime}(\bar{t})$. For steep roughness (figure 6) we have both $\bar{d}_{0}^{\prime}(\bar{t}) \rightarrow \pm \infty$ and multivaluedness, both initially identified as criteria for a touchdown by [57]. The effect of ice accretion is intriguing since it seems able to both increase (mild figure 4 ) and reduce (marginal figure 8 ) the local maximum in the contact speed $\bar{d}_{0}^{\prime}(\bar{t})$, the latter reinforcing the above suggestion that icing helps avoid a touchdown. This confirms that newly formed ice can act as roughness does, bearing in mind that the present roughness has a positive height. The splash-jet solution (4.25) is represented by the plots of the characteristic traces given in figures 10 and 11 for the mild and steep roughness cases with ice accretion. Clashings and crossings which are clear in the two figures are an important extra feature that depends somewhat on the value of $K$ and hence on the ice-growth rate $\gamma$ of (3.3) and density ratio $\rho$. Even for the mild slope the change in $\bar{d}_{0}^{\prime}(\bar{t})$ causes characteristic traces which initially fan out from the data curve $\bar{t}=\tau(\bar{x})$, but subsequently converge and intersect one another. (See comments on splash and jets in section 1). This indicates the presence of a hydraulic jump. Similar comments hold with or without ice accretion and for the case of a steep slope, where additionally we have characteristic traces re-intersecting the data curve, which likewise might be regarded as a criterion for touchdown. These all indicate that the inviscid splash jet (4.25) must then be altered, causing (if nothing else) some further restructuring of the viscous sublayer beneath it. Physically one main repercussion here for the more severe roughnesses is that jumps or sudden movements of the contact point, of the ice shapes and of the free surface are likely to take place under certain conditions. The normal momentum of the original outermost flow is converted by impact into tangential momentum which is sufficient to sweep the droplet over many roughnesses with or without new icing. This sweep effect led by the moving contact point may in certain cases be hindered or partially blocked by some existing roughness shapes or pre-existing ice but apart from changes in the splash-jet dynamics which may be a secondary influence altogether that blockage attempt can be counteracted by jumps of the contact-point movement due to the strength of the tangential momentum.

\section{The inner layer responses}

The outer interactions found in the previous two sections determine the quantities of most significance to the overall properties of the impact flows with 

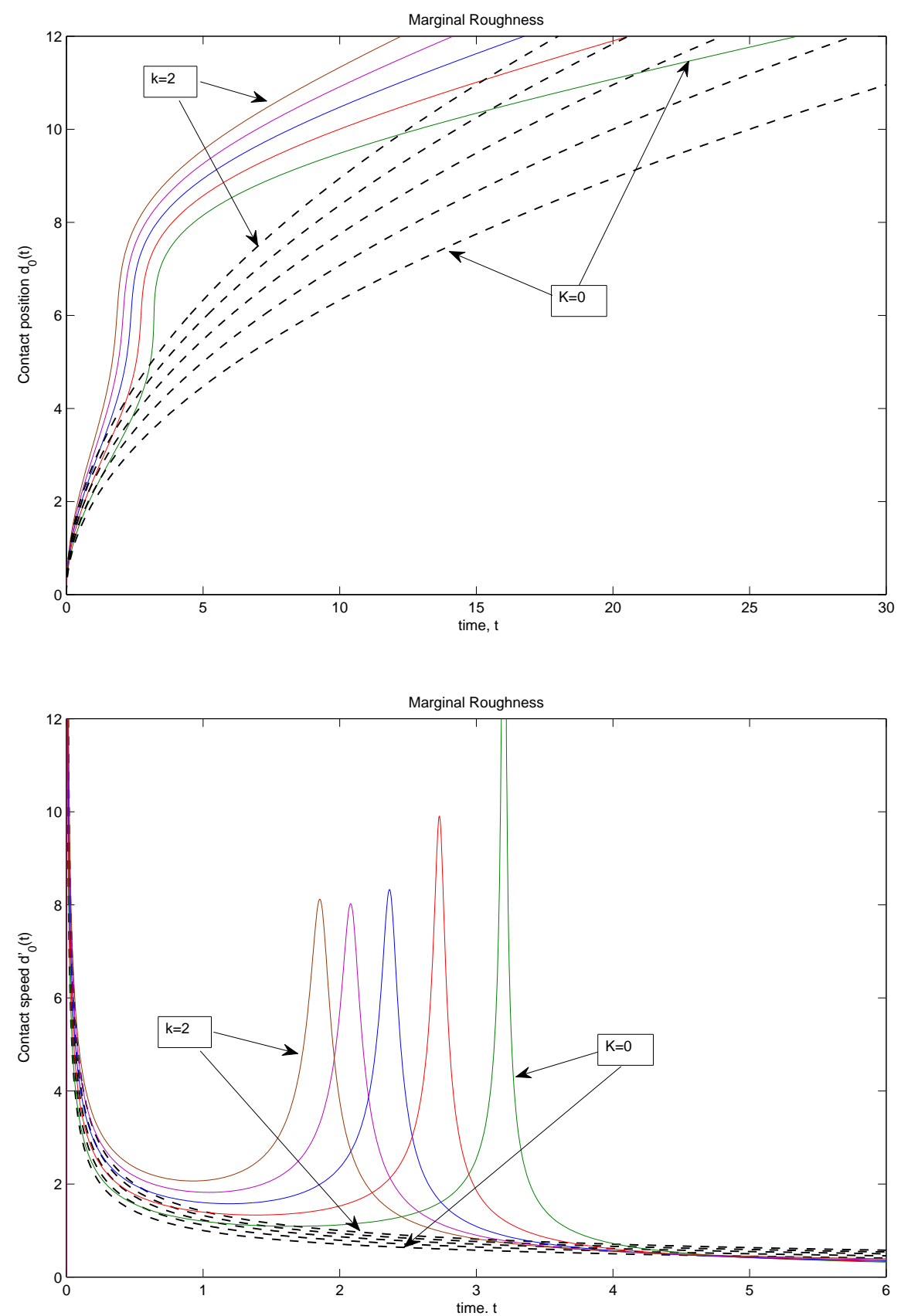

Figure 8 . The contact point position $\bar{d}_{0}(\bar{t})$ and speed $\bar{d}_{0}^{\prime}(\bar{t})$ for a marginal surface roughness, with $K=(1-\rho) \gamma=0,0.5,1.0,1.5,2.0$. The dashed curves denote the analytic form (4.8a) without roughness. The onset of breakdown is apparent in the plots on the right. 

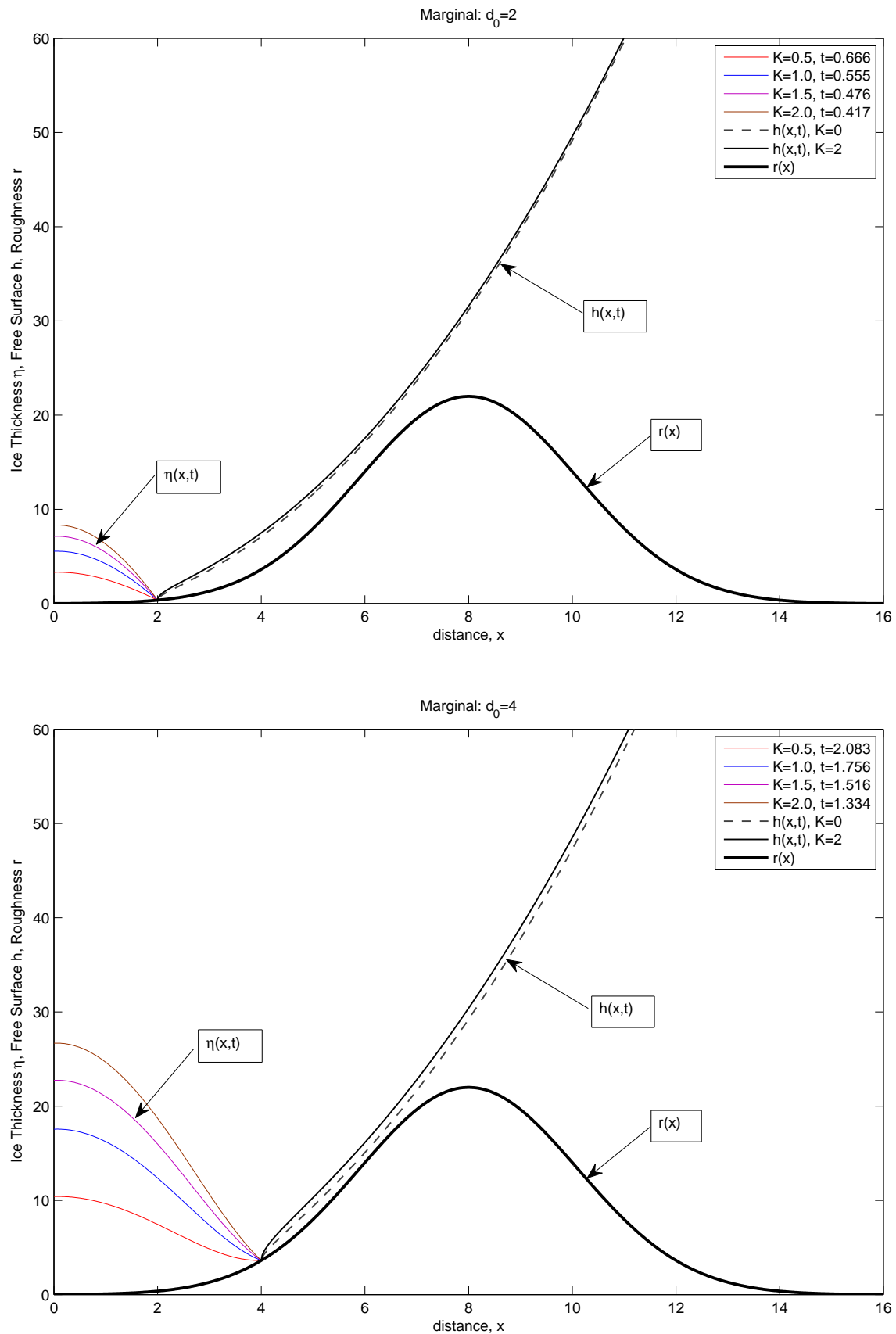

Figure 9. The ice thickness $\bar{\eta}$, free surface shape $\bar{h}$ and marginal surface roughness $\bar{r}$ as in figure 8 , when $\bar{d}_{0}=2,4,6$ and 8 , for $K=(1-\rho) \gamma=0,0.5,1,1.5$ and 2 . The free surface is seen to lie tangentially on the rough surface at $\bar{d}_{0}=4$, only for $K=(1-\rho) \gamma=0$. 

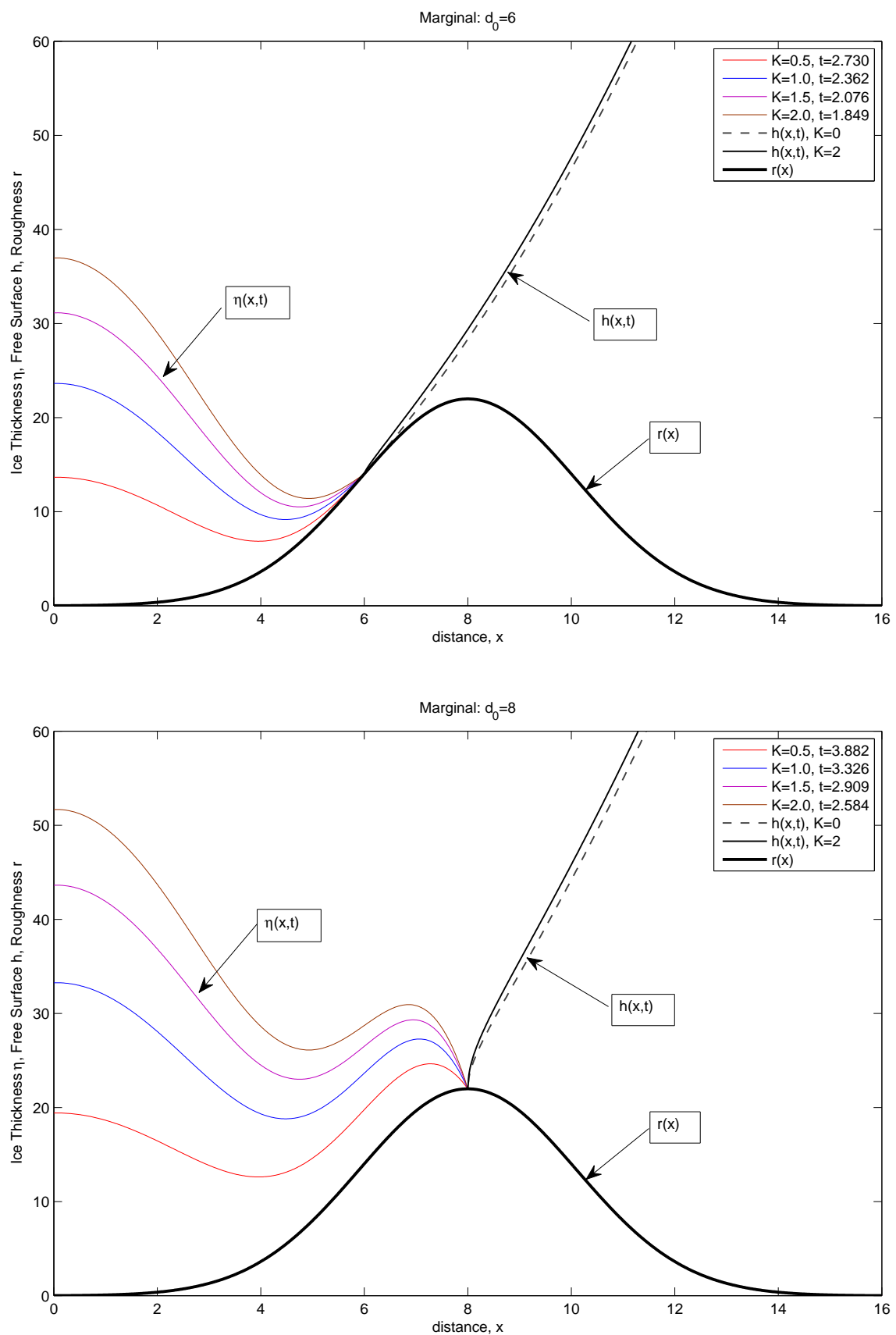

Figure 9. The ice thickness $\bar{\eta}$, free surface shape $\bar{h}$ and marginal surface roughness $\bar{r}$ as in figure 8 , when $\bar{d}_{0}=2,4,6$ and 8 , for $K=(1-\rho) \gamma=0,0.5,1,1.5$ and 2 . The free surface is seen to lie tangentially on the rough surface at $\bar{d}_{0}=4$, only for $K=(1-\rho) \gamma=0$. 


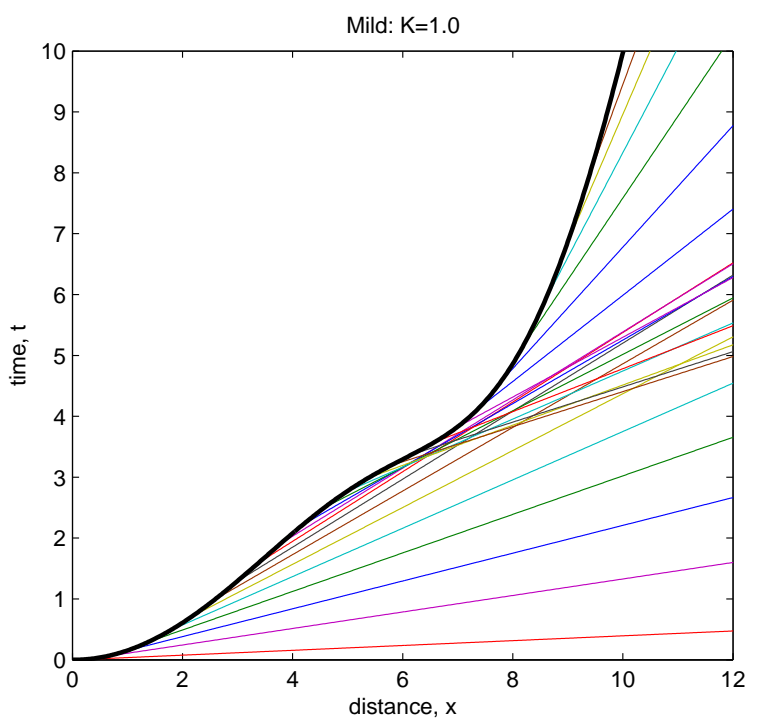

Figure 10. Characteristic curves for the splash jet for a shallow surface roughness with ice accretion as in figures 4,5 , for $K=(1-\rho) \gamma=1.0$.

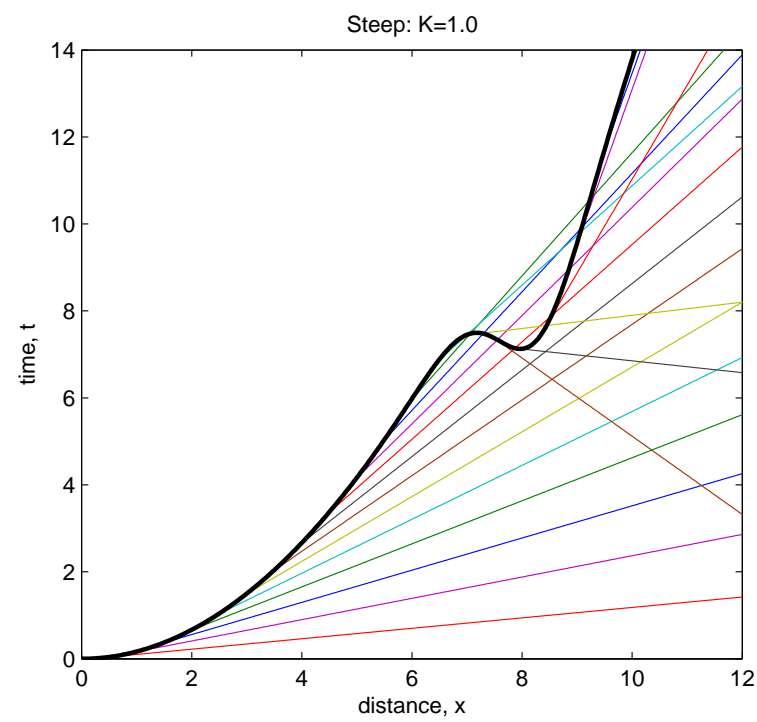

Figure 11. Characteristic curves for the splash jet for a steep surface roughness, with ice accretion as in figures 6,7 , for $K=(1-\rho) \gamma=1.0$. 
solidification and comparing with experiment as will be shown in the next section. The inner near-wall effects touched on in $\S 4$ are also of much interest in terms of completing the multi-structure of figure 2 especially near the foot of the moving contact point as well as demonstrating the likely restricted region of turbulence (see references in §3) and details of these are available from the authors on request.

In particular matching confirms the viscous flow very near the turnover position to be a combination of most likely separating, abruptly transitional and turbulent in reality. This result is mitigated however by the fact that the moving contact point sweeps away the turbulent flow to leave behind a laminar structure. The section below accordingly tends to concentrate on overall effects produced by the outer interactions.

\section{Experimental comparisons and closing remarks}

Comparisons with experiments and the links with a body of experimental and engineering work along with practical findings are investigated in $\$ 7.1$ below followed in $\$ 7.2$ by concluding remarks.

\subsection{COMPARISONS WITH EXPERIMENTS}

The body of relevant experimental and engineering work provided in the comprehensive [70] report enables connections to be made between practical results and our investigation; see also other interesting experimental aspects in [5], [31], [7], [6]. [70] includes some smaller-scale data in addition to larger-scale data on ice accretion. Thus the droplet diameters of 15-100 microns or more are typically of concern according to [81] (page 1-3 of [70]) as in the present study, while standard values of 20 microns and a maximum value of 50 microns are quoted by [82] (page 3-27) again in keeping with the present work. The contribution [82] is mostly concerned with ice shapes and their effects on aerodynamics and safety and it also (page 3-31) highlights the appropriate ranges of temperatures as $32 \mathrm{~F}$ to $-40 \mathrm{~F}$, or $0 \mathrm{C}$ to $-15 \mathrm{C}$, confirming the range given in our introduction, while it shows (pages 3-8, 3-11) an average icing rate of $97 \mathrm{~mm} /$ hour which given the different operative conditions is not far quantitatively from the prediction range presented earlier in this subsection. Interesting effects of temperature and droplet size are shown on pages $3-15,3-16$, and the use of roughness is confirmed as a surrogate for built-up icing on page 3-19. Later in the report [83] (pages 4-4,4-8,4-9) indicate parameters for example on time scales with which the theory here connects, whereas the point that ice roughness is very significant is demonstrated on pages 4-5,4-16. [84] show further useful parameters numerically (page 5-8) and in particular graphs of results of accreted roughness versus 
time (page 5-10, 5-11) which are fairly close to our specific predictions as described next. (Encouragement for further research of this kind is seen on pages 5-12, 5-13 of the same contribution.)

Comparisons are made in our figure 12 between the current theoretical predictions and the specific case of [85], [35] which is highlighted in figure 518 of [70]. The comparisons take into account the fact that $S t$ tends to be less than unity in most aircraft icing settings. For the representative single droplet the theory predicts approximately as an interpretation that the ice spreads in the horizontal direction $x^{*}$ to about $\sqrt{2}$ times the droplet diameter $L^{*}$ (which is $2 R_{D}^{*}$ ) as a typical value and spreads vertically in $y^{*}$ to a height $H^{*}$ of at least $R e^{-1 / 2} L^{*}$ for a first approximation which may be a lower bound. The preexisting surface roughness or ice due to previous droplets perhaps reduces the $\sqrt{2}$ factor to unity while the duration of the process may be taken as $L^{*} / U_{D}^{*}$, the characteristic droplet travel time. Working with the above and considering the above figure we take 20 microns for $L^{*}$ and the value $10^{4}$ for $R e$. Then one droplet yields a height $H^{*}$ of $10^{-2} \times 20 / 10^{6} \mathrm{~m}$, that is $2 \times 10^{-7} \mathrm{~m}$. (A typical $1 \mathrm{~m}$ value for the spanwise distance scale is taken where necessary.) Moreover the low liquid water content LWC of $0.5 \mathrm{~g} / \mathrm{m}^{3}$ implies in broad terms many droplets in the $\mathrm{m}^{3}$ cube: say about 2,000 (if we allow for 20-40 micron drop diameters). On the other hand those that land on a specific piece of existing ice do so with a small probability, of only about $R e^{-1 / 2}$ say, from comparing the ice thickness with the cube thickness and attempting to adjust for oblique impacts. So that probability is about $10^{-2}$. Hence only around 20 of the droplets actually impact on the existing ice piece per second. Impacts are thus say 20 droplets per second at an incident speed of $150 \mathrm{mph}$, i.e. about $50 \mathrm{~m} / \mathrm{s}$, while allowing for a free-stream speed as distinct from the near-stagnation speed near the front of the body in the experiments. See also next-but-one paragraph. Hence a height $H^{*}$ is suggested as $20 \times$ $2 / 10^{7} \mathrm{~m}$, in a second, leading to the vertical accretion velocity being around $4 \times 10^{-6} \mathrm{~m} / \mathrm{s}$. This gives an approximate estimate of $0.25 \mathrm{~mm} / \mathrm{min}$. (A lower Reynolds number of $10^{3}$ would reduce the estimate by a multiplicative factor of $1 / \sqrt{10}$ and a doubling of the droplet diameter would increase the estimate by a multiplicative factor of 2.) That value lies well within the experimental range for AGARD's figure 5-18 as the present figure 12 indicates by means of three lines marked A, B, C which represent the estimate of $0.25 \mathrm{~mm} / \mathrm{min}$ and the influences of changing $R e$ and droplet size respectively.

Various factors may increase or decrease the estimate. There are also many limitations to acknowledge of course and so the comparisons are made tentatively. The theoretical details explored so far here have the time $t^{*}$ assumed comparatively small which is observed in figure 12 to be when the experimental and theoretical trends do agree most whereas predictions for later times must be regarded as extrapolations; numerous real effects as mentioned in the paper are neglected; the practical situations are very complex as 


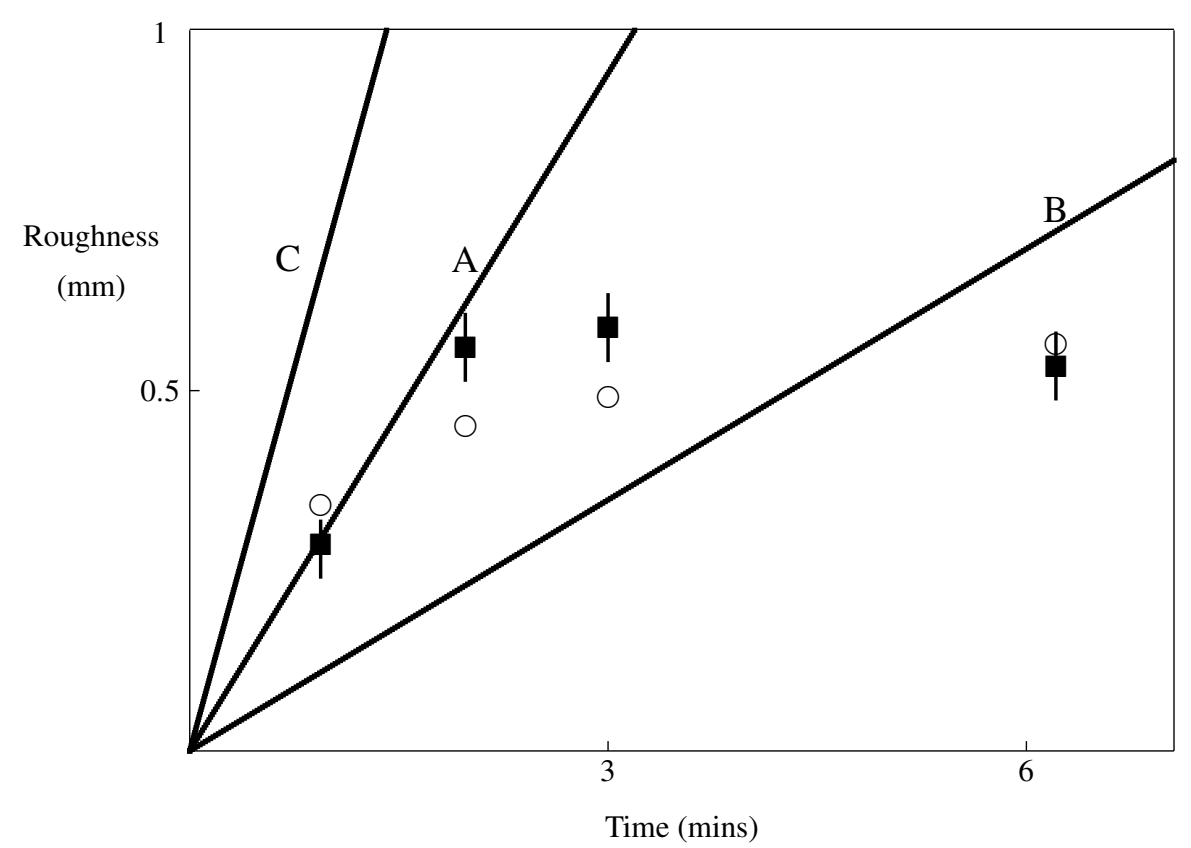

Figure 12. Ice thickness vs time. Comparisons between present predictions (thick solid lines $\mathrm{A}, \mathrm{B}, \mathrm{C})$ for small times and experimental measurements (black squares with error bars shown) and engineering modelling (open circles) of [85], [35]. Case B is obtained from base case A by increasing the Reynolds number while case $\mathrm{C}$ corresponds to increased droplet size.

[70] makes clear. Influences in the surrounding air motion, the surface tension and pre-layers of water are neglected. Above all perhaps is the feature that the model is a two-dimensional one dealing with the flow-solidification interplay for only a single droplet. By contrast on the positive side the theory is built from first principles, it is analytic predominantly and certain major aspects are included, with the effects of many droplets freezing being attributed to added surface roughness.

\subsection{Closing comments}

The present theoretical study potentially opens up several new avenues of research in important application areas and the comparison in figure 12 attests to the potential practical value. Also the proportion of the droplet liquid volume frozen during the small-time impact behaviour of a single droplet can be predicted, as $\mathrm{O}\left(R e^{-\frac{1}{2}}\right)$ or approximately $1 \%$, and the asymptotic approach and comparisons confirm the relevance of a 1-D phase-change problem extended over many droplet impacts. In aircraft-icing super-cooled water droplets impact on the wing when it flies through certain freezing conditions. These droplets can freeze immediately or gradually on impact and cause ice 
structures to form on the aircraft. This in turn can result in a loss of lift and an increase in the drag, and, in the extreme case, may lead to the crashing of the aircraft, with potential fatalities.

The major result of this study, supplementary to the finding of plausible agreement with experiments and engineering models in \$7.1 and figure 12, has been to incorporate ice growth systematically into a model soon after the impact of a supercooled droplet onto a solid surface. In addition we have combined its effect with surface roughness produced by previous impacts for instance, subject to fairly reasonable assumptions on the ranges of the Reynolds, Weber, Stefan numbers and other parameters. Without any surface roughness the influence of icing can be to increase or decrease the spreading of the droplet. Given that the major influence of surface roughness alone is to slow the spreading of a droplet as in [57], the combination of the two effects is likely to be difficult to immediately deduce. It may be whilst surface roughness acts to keep the droplet concentrated in a perhaps protected region, the ice growth can work against this, leading to the propulsion of unfrozen water to unprotected areas.

An extra facet is the incorporation of the effects of viscosity into a droplet model which traditionally has in the main been described using a purely inviscid description. This in itself has led to subtleties regarding the existence of laminar viscous-flow solutions, which leads on to the inference described in $\$ 3$ of turbulent or semi-turbulent pre-flow. However it is seen that the effect of the moving contact point is to sweep away the limited area of transition to turbulent flow downstream and ensure the laminar analysis remains valid. The splash jet preceding the contact point is pushed ahead and is decelerating, which physically tends to induce turbulent flow there, while the follow-up viscous layer is pulled along by the dominant momentum of the jet-root region. Another extra facet to be explored concerns the substantial changes observed here within the splash jet in the presence of icing with or without wall roughness. These changes are able to induce hydraulic jumps. The alternative of comparable ice accretion on the wet and dry sides of the contact point should also be noted at this point. Further mention should be made of one limitation of the current work which is that the results are rather limited to special cases as a means of demonstrating the major features described above. Clearly any cases where the condition (4.5) for a single contact point is not satisfied and so possible multiple contact points or multi-patches occur need to be investigated much further. Thus the current analysis and numerical results are for droplet impacts on a rough surface with the added complication of icing, for certain cases only. The icing spread alone can be much faster than the $2 \sqrt{t}$ law found in the absence of icing while the influence of surface roughness then works against such a simple description. The present study is intended to help quantify the two opposing effects; the 
comparisons and links described in $§ 7.1$ seem to give the present approach quite substantial support.

\section{Acknowledgements}

Thanks are due to the UK Icing Group, especially Roger Gent and David Hammond for their insights, and to the referees for their helpful comments.

\section{References}

1. Stan CA, Schneider GF, Shevkoplyas SS, Hashimoto M, Ibanescu M, Wiley BJ, Whitesides GM (2009) A microfluidic apparatus for the study of ice nucleation in supercooled water drops. Lab Chip 9:2293-2305

2. Mason BJ (1976) The physics of clouds. Oxford Monographs on Meterology, 2nd Edn. 594. Oxford: Clarendon

3. Gent RW, Dart NP, Cansdale JT (2000) Aircraft Icing. Phil Trans R Soc Ser A 358:28732911

4. Lynch FT, Khodadoust A (2001) Effects on ice accretions on aircraft aerodynamics. Progress Aerospace Sci 37:669-767

5. Hindmarsh JP, Russell AB, Chen XD (2003) Experimental and numerical analysis of the temperature transition of a suspended freezing water droplet. Int J Heat Mass Trans 46:1199-1213

6. Jung S, Tiwari MK, Doan NV, Poulikakos D (2012) Mechanism of supercooled droplet freezing on surfaces. Nature Commun 3:615-623

7. Zheng L, Li Z, Bourdo S, Khedir KR, Asar MP, Ryerson CC, Biris AS (2011) Exceptional superhydrophobicity and low velocity impact icephobicity of acetonefunctionalized carbon nanotube films. Langmuir 27:9936-9943

8. Worthington AM (1876) On the form assumed by drops of liquids falling vertically on a horizontal plate. Proc R Soc Ser A 283:141-173

9. Edgerton HE, Killian JR (1939) Flash Boston: Branford.

10. Rioboo R, Marengo M, Tropea C (2002) Time evolution of a liquid drop impact onto solid, dry surfaces. Exp Fluids 33:112-124

11. Rioboo R, Tropea C, Marengo M (2001) Outcomes from a drop impact on solid surfaces. Atomization and Sprays 33:155-165

12. $\breve{S i k a l o ~} \breve{S}$, Tropea C, Ganić EN (2005) Impact of droplets onto inclined surfaces. J Colloid Interface Sci 286:661-669

13. Rein M (1993) Phenomena of liquid drop impact on solid and liquid surfaces. Fluid Dynamics Research 12:61-93

14. Yarin AL (2006) Drop impact dynamics: splashing, spreading, receding, bouncing. Ann. Rev. Fluid Mech 38:159-192

15. Engel OG (1955) Water drop collisions with a solid surface. J Res Nat Bur Stand 54:281298

16. Jones H (1973) Splat cooling and metastable phases. Rep Prog Phys 36:1425 [see also Jones H (1982) Rapid solidification of metals and alloys, Monograph 8, Institute of Metallurgists, London.] 
17. Collings EW, Markworth AJ, McCoy JK, Saunders JH (1990) Splat-quench solidification of freely falling liquid-metal drops by impact on a planar substrate. J Mat Sci 23:3677-3682

18. Chandra S, Avedisian, CT (1991) On the collision of a droplet with a solid surface. Proc R Soc Ser A 432:13

19. Pasandideh-Fard M, Qiao YM, Chandra S, Mostaghimi J (1996) Capillary effects during droplet impact on a solid surface. Phys Fluids 8:650-659

20. Roisman IV, Rioboo R, Tropea C (2002) Normal impact of a liquid drop on a dry surface: model for spreading and receding. Proc R Soc Ser A 458:1411-1430

21. Roisman IV (2009) Inertia dominated drop collisions. II An analytic solution of the Navier-Stokes equations for a spreading viscous film. Phys Fluids 21:052104

22. Roisman IV, Berberovic E, Tropea C (2009) Inertia dominated drop collisions. I On the universal flow in the lamella. Phys Fluids 21:052103

23. Lagubeau G, Fontelos MA, Josserand C, Maurel A, Pagneux V, Petitjeans P (2012) Spreading dynamics of drop impacts. J Fluid Mech 713:50-60

24. Roisman IV (2010) Fast forced liquid film spreading on a substrate: flow, heat transfer and phase transition. J Fluid Mech 656:189-204

25. Madjeski J (1976) Solidification of droplets on a cold surface. Int J Heat Mass Transfer 19:1009-1013

26. Madjeski J (1983) Droplets on impact with a solid surface. Int J Heat Mass Transfer 26:1095-1098

27. Bennett T, Poulikakos D (1993) Splat-quench solidification: estimating the maximum spreading of a droplet impacting a solid surface. J Mat Sci 28:963-970

28. Bennett T, Poulikakos D (1994) Heat transfer aspects of splat-quench solidification: modelling and experiment. J Mat Sci 29:2025-2039

29. Evans PV, Greer AL (1988) Modelling of crystal growth and solute redistribution during rapid solidification. J Mater Sci Engng 98:357

30. Cebeci T (1989) Calculation of flow over iced airfoils. AIAA J 27:853-861

31. Fortin G, Laforte JL, Ilinca A (2006) Heat and mass transfer during ice accretion on aircraft wings with an improved roughness model. Int J Thermal Sci 45:595-606

32. Collyer MR (1976) A user guide to a program for computing viscous transonic flows past aerfoils. RAE Tech Memo Aero 1693, September 1976

33. Messinger BL (1953) Equilibrium temperature of an unheated icing surface as a function of air speed. J Aero Sci 29-43

34. Messinger BL (1953) Equilibrium temperature of an unheated icing surface as a function of air speed. J Aero Sci 29-43

35. Wright WB, Bidwell CS (1995) Additional improvements to the NASA lewis ice accretion code LEWICE, NASA TM-106849, AIAA-95-0752

36. Brakel TW, Charpin JPF, Myers TG (2007) One-dimensional ice growth due to incoming supercooled droplets impacting on a thin conducting substrate. Int J Heat Mass Transfer 50:1694-1705

37. Myers TG, Charpin JPF, Chapman SJ (2002) The flow and solidification of a thin fluid film on an arbitrary three-dimensional surface. Phys Fluids 14:2788-2803

38. Myers TG, Charpin JPF, Thompson CP (2002) Slowly accreting ice due to supercooled water impacting on a cold surface. Phys Fluids 14:240-256

39. Myers TG, Charpin JPF (2004) A mathematical model for atmospheric ice accretion and water flow on a cold surface. Int J Heat Mass Transfer 47:5483-5500

40. Myers TG, Hammond DW (1999) Ice and water film growth from incoming supercooled droplets. Int. J Heat Mass Transfer 42:2233-2242

41. Tsao JC, Rothmayer AP (2002) Application of triple-deck theory to the prediction of glaze ice roughness formation on an airfoil leading edge. Comput. Fluids 31:977-1014 
42. Quero M, Hammond DW, Purvis R, Smith FT (2006) Analysis of supercooled water impact on a thin water layer and ice growth. AIAA Paper 466:5536-5548

43. Wagner H (1932) Uber stoss-und gleitvorgänge an der oberfläche von flüsigkeiten (phenomena associated with impacts and sliding on liquid surfaces). Zeitschrift fur Angewandte Mathematik und Mechanik 12:193-215

44. Bowden FP, Field JE (1964) The brittle fracture of solids by liquid impact, by solid impact, and by shock. Proc R Soc Ser A 260:94-95

45. Lesser MB, Field JE (1983) The impact of compressible liquids. Ann Rev Fluid Mech 15:97-122

46. Mandre S, Brenner MP (2012) The mechanism of a splash on a dry solid surface. J Fluid Mech 690:148-172

47. Howison SD, Ockendon JR, Oliver JM (2002) Deep- and shallow-water slamming at small and zero deadrise angles. J Engng Maths 42:373-388

48. Howison SD, Ockendon JR, Wilson SK (1991) Incompressible water-entry problems at small deadrise angles. J Fluid Mech 222:215-230

49. Oliver JM (2002) Water entry and related problems. D. Phil. Thesis, Univ of Oxford

50. Korobkin AA (1996) Advances in Marine Hydrodynamics. Ch. 7, Water impact problems in ship hydrodynamics. Computational Mechanics Publications 323-339

51. Korobkin AA (1997) Asymptotic theory of liquid-solid impact. Phil Trans R Soc Ser A 355:507-522

52. Korobkin AA (1999) Shallow water impact problems. J Engng Maths 35:233-250

53. Howison SD, Ockendon JR, Oliver JM, Purvis R, Smith FT (2005) Droplet impact on a thin fluid layer. J Fluid Mech 542:1-23

54. Korobkin AA, Ellis AS, Smith FT (2008) Trapping of air in impact between a body and shallow water. J Fluid Mech 611:365-394

55. Purvis R, Smith FT (2004) Air-water interactions near droplet impact. Eur J Appl Maths 15:853-871

56. Purvis R, Smith FT (2005) Droplet impact water layers: post-impact analysis and computations. Phil Trans R Soc Ser A 611:1209-1221

57. Ellis AS, Smith FT, White AH (2011) Droplet impact on to a rough surface. Quart J Mech Appl Math 64:1-37

58. Hicks PD, Smith FT (2011) Skimming impacts and rebounds on shallow liquid layers. Proc R Soc Ser A 467:653-674

59. Carslaw HS, Jaeger JC (1959) Conduction of Heat in Solids. Oxford University Press, Oxford.

60. Crank J (1984) Free and Moving Boundary Problems. Oxford University Press, Oxford.

61. Worster MG (2000) Interfacial Fluid Dynamics, Ch 8 of Perspectives in Fluid Dynamics (Ed Batchelor GK, Moffat HK, Worster MG) Cambridge.

62. Löwen H, Bechhoefer J, Tuckerman LS (1992) Crystal growth at long times: Critical behaviour at the crossover from diffusion to kinetics-limited regimes. Physical Review A 45:2399-2415

63. Howison SD, Ockendon JR, Lacey AA (1985) Singularity development in moving boundary problems. Quart J Mech Appl Maths 14:343-360

64. Dewynne JN, Howison SD, Ockendon JR, Xie W (1989) Asymptotic behaviour of solutions to the Stefan problem with a kinetic condition at a free boundary. J Austral Math Soc B 31:81-96

65. Umantsev A, Davis SH (1992) Growth from a hypercooled melt near absolute stability. Physical Review A 45:7195-7201

66. Charach Ch, Zaltzman B (1993) Planar solidification from an undercooled melt: Asymptotic solutions to a continuum model with interfacial kinetics. Physical Review E 47:1230-1234 
67. Charach Ch, Zaltzman B (1994) Analytic model for planar growth of a solid germ from an undercooled melt. Physical Review E 49:4322-4327

68. Evans JD, King JR (2000) Asymptotic results for the Stefan problem with kinetic undercooling. Quart J Mech Appl Math 53:449-473

69. Evans JD, King JR (2003) The Stefan problem with nonlinear kinetic undercooling. Quart J Mech Appl Math 56:139-161

70. AGARD (1997) Ice Accretion Simulation. Report of the fluid Dynamics Working Group 20, AGARD Advisory Report, AGARD-AR-344, ISBN 92-836-1067-9

71. Davis SH (2000) Interfacial Fluid Dynamics, Ch 1 of Perspectives in Fluid Dynamics (Ed Batchelor GK, Moffat HK, Worster MG) Cambridge.

72. Klemp JB, Acrivos A (1976) A moving-wall boundary layer with reverse flow. J Fluid Mech 70:363-381

73. Weidman PD, Kubitschek DG, Davis AMJ (2006) The effect of transpiration on selfsimilar boundary-layer flow over moving surfaces. Int J Engng Sci 44:730-737

74. Sychev VV (1980) On certain singularities in solutions of equations of boundary layer on a moving surface. P M M Soc USSR 44:587

75. Elliott JW, Cowley SJ, Smith FT (1983) Breakdown of boundary layers; (i) on moving surfaces; (ii) in semi-similar unsteady flow; (iii) in fully unsteady flow. Geophys Astrophys Fluid Dyn 25:77-138

76. Degani AT, Walker JD, Smith FT (1998) Unsteady separation past moving surfaces. J Fluid Mech 375:1-38

77. Smith FT, Elliott JW (1985) On the abrupt turbulent reattachment downstream of leading-edge laminar separation. Proc R Soc Ser A 401:1-27

78. Braun S, Kluwick A (2005) Blow-up and control of marginally separated boundary layers. Phil Trans R Soc Ser A 363:1057-1067

79. Bowles RI, Davies C, Smith FT (2003) On the spiking stages in deep transition and unsteady separation. J Eng Math 45:227-245

80. Scheichl B, Kluwick A, Smith FT (2011) Break-away separation for high turbulence intensity and large Reynolds number. J Fluid Mech 670:260-300

81. Wagner B, Hammond D, van Hengst J, Gent R, Kind R (1997) Introduction, in Ice Accretion Simulation. Report of the fluid Dynamics Working Group 20, AGARD Advisory Report AGARD-AR-344, ISBN 92-836-1067-9

82. van Hengst J, Gent R, Hammond D, Wagner B (1997) Ice accretion and its effects on aircraft, in Ice Accretion Simulation. Report of the fluid Dynamics Working Group 20, AGARD Advisory Report AGARD-AR-344, ISBN 92-836-1067-9

83. King R, Feo A, Golia C, Shah A (1997) Experimental techniques and facilities, in Ice Accretion Simulation. Report of the fluid Dynamics Working Group 20, AGARD Advisory Report AGARD-AR-344, ISBN 92-836-1067-9

84. Potapczuk MG, Gent R, Gifford D (1997) Review, validation and extension of ice accretion prediction codes, in Ice Accretion Simulation. Report of the fluid Dynamics Working Group 20, AGARD Advisory Report AGARD-AR-344, ISBN 92-836-1067-9

85. Shin J (1994) Characteristics of surface roughness associated with leading edge ice accretion. AIAA-94-0799 [See also J Aircraft (1996) 33:316-321]

Address for Offprints:

KLUWER ACADEMIC PUBLISHERS PrePress Department,

P.O. Box 17, 3300 AA Dordrecht, The Netherlands

e-mail: TEXHELP@WKAP.NL

Fax: +31786392500 